\title{
The intergalactic magnetic field probed by a giant radio galaxy
}

\author{
S. P. O’Sullivan ${ }^{1}$, J. Machalski ${ }^{2}$, C. L. Van Eck ${ }^{3}$, G. Heald ${ }^{4}$, M. Brüggen ${ }^{1}$, J. P. U. Fynbo ${ }^{5}$, K. E. Heintz ${ }^{6}$,
} M. A. Lara-Lopez ${ }^{7}$, V. Vacca ${ }^{8}$, M. J. Hardcastle ${ }^{9}$, T. W. Shimwell ${ }^{10,11}$, C. Tasse ${ }^{12,13}$, F. Vazza ${ }^{14,1,19}$, H. Andernach ${ }^{15}$, M. Birkinshaw ${ }^{16}$, M. Haverkorn ${ }^{17}$, C. Horellou ${ }^{18}$, W. L. Williams ${ }^{9}$, J. J. Harwood ${ }^{9}$, G. Brunetti ${ }^{19}$, J. M. Anderson ${ }^{20}$, S. A. $\mathrm{Mao}^{21}$, B. Nikiel-Wroczyński ${ }^{2}$, K. Takahashi ${ }^{22}$, E. Carretti ${ }^{19}$, T. Vernstrom ${ }^{23}$, R. J. van Weeren ${ }^{11}$, E. Orrú ${ }^{10}$, L. K. Morabito ${ }^{24}$, and J. R. Callingham ${ }^{10}$

(Affiliations can be found after the references)

Received 12 July 2018 / Accepted 9 October 2018

\begin{abstract}
Cosmological simulations predict that an intergalactic magnetic field (IGMF) pervades the large scale structure (LSS) of the Universe. Measuring the IGMF is important to determine its origin (i.e. primordial or otherwise). Using data from the LOFAR Two Metre Sky Survey (LoTSS), we present the Faraday rotation measure (RM) and depolarisation properties of the giant radio galaxy $\mathrm{J} 1235+5317$, at a redshift of $z=0.34$ and $3.38 \mathrm{Mpc}$ in size. We find a mean RM difference between the lobes of $2.5 \pm 0.1 \mathrm{rad} \mathrm{m}^{-2}$, in addition to small scale $\mathrm{RM}$ variations of $\sim 0.1 \mathrm{rad} \mathrm{m} \mathrm{m}^{-2}$. From a catalogue of LSS filaments based on optical spectroscopic observations in the local universe, we find an excess of filaments intersecting the line of sight to only one of the lobes. Associating the entire RM difference to these LSS filaments leads to a gas density-weighted IGMF strength of $\sim 0.3 \mu \mathrm{G}$. However, direct comparison with cosmological simulations of the RM contribution from LSS filaments gives a low probability $(\sim 5 \%)$ for an RM contribution as large as $2.5 \mathrm{rad} \mathrm{m}^{-2}$, for the case of IGMF strengths of $10-50 \mathrm{nG}$. It is likely that variations in the RM from the Milky Way (on $11^{\prime}$ scales) contribute significantly to the mean RM difference, and a denser RM grid is required to better constrain this contribution. In general, this work demonstrates the potential of the LOFAR telescope to probe the weak signature of the IGMF. Future studies, with thousands of sources with high accuracy RMs from LoTSS, will enable more stringent constraints on the nature of the IGMF.
\end{abstract}

Key words. galaxies: active - radio continuum: galaxies - galaxies: magnetic fields - galaxies: jets - techniques: polarimetric galaxies: clusters: individual: J1235+5317

\section{Introduction}

Diffuse gas is expected to permeate the large-scale structure (LSS) of the Universe away from galaxy groups and clusters. Detecting and characterising this intergalactic gas is challenging due to the expected low particle number density $\left(\sim 10^{-5}-10^{-6} \mathrm{~cm}^{-3}\right)$ and temperature $\left(10^{5}-10^{7} \mathrm{~K}\right)$. Although diffuse, this warm-hot intergalactic medium (WHIM; Davé et al. 2001; Cen \& Ostriker 2006) potentially contains half the total baryon content of the local Universe (Bregman 2007; Nicastro et al. 2018). In addition, accretion shocks along these LSS filaments are predicted to accelerate particles to relativistic energies and to amplify magnetic fields. Thus, detecting this filamentary structure in synchrotron emission using radio telescopes is a promising avenue for studying the WHIM (e.g. Vazza et al. 2015a). Recent statistical studies based on the cross-correlation of diffuse radio synchrotron emission and the underlying galaxy distribution have derived upper limits on the magnetisation of filaments of the order of $0.1 \mu \mathrm{G}$ (Vernstrom et al. 2017; Brown et al. 2017). Furthermore, Vacca et al. (2018) found a faint population of sources which might be the tip of the iceberg of a class of diffuse large-scale synchrotron sources associated with the WHIM connected to a large-scale filament of the cosmic web. An alternative approach is to measure the Faraday rotation properties of the magnetised WHIM using many bright, polarised, background radio sources (e.g. Stasyszyn et al. 2010; Akahori et al. 2014; Vacca et al. 2016).

From simulations, the field strength of the intergalactic magnetic field (IGMF) is expected to be in the range of $1-100 \mathrm{nG}$ (e.g. Dolag et al. 1999; Brüggen et al. 2005; Ryu et al. 2008; Vazza et al. 2017). It is important to constrain the magnetic field in the WHIM in order to determine the unknown origin of the large scale magnetic field in the Universe (Zweibel 2006). While large scale fields are commonly detected in galaxies and galaxy clusters, the strong modification of these fields erases the signature of their origin (e.g. Vazza et al. 2015b). This may not be the case in the WHIM, as the amplification of primordial magnetic fields in these filamentary regions are likely primarily due to compressive and shearing gas motions, in addition to small-scale shocks, such that the observed level of magnetisation could be connected to the seeding process (e.g. Ryu et al. 2008; Vazza et al. 2014). The AGN and star formation activity in galaxies can also drive powerful outflows that may significantly magnetise the intergalactic medium on large scales (e.g. Furlanetto \& Loeb 2001; Donnert et al. 2009; Beck et al. 2013). Therefore, distinguishing between a primordial origin and a later injection of magnetic field that was initially generated on smaller scales by galaxies and stars is a key goal for studies of the IGMF (see Akahori et al. 2018, and references therein).

It has also been proposed to study the WHIM using large or "giant" radio galaxies (GRGs) whose linear size can extend beyond $1 \mathrm{Mpc}$, with the largest such example being $4.7 \mathrm{Mpc}$ in extent (Machalski et al. 2008). GRGs are usually FRII type radio galaxies (e.g. Dabhade et al. 2017), although some giant FRI also exist (e.g. Heesen et al. 2018; Horellou et al. 2018), that extend well beyond their host galaxy and local environments, into the surrounding intergalactic medium. Asymmetries in the GRG morphology can be used as a probe of the ambient 
gas density (Subrahmanyan et al. 2008; Safouris et al. 2009; Pirya et al. 2012; Malarecki et al. 2015) and the Faraday rotation properties of the polarised emission from the lobes can be used to study the magnetic field properties of the surrounding gas on Mpc scales (Xu et al. 2006; O'Sullivan et al. 2018). Another potential approach to studying the magnetised WHIM in cluster outskirts is by using Faraday rotation observations of the highly polarised emission from radio relics (e.g. Kierdorf et al. 2017; Loi et al. 2017).

The effect of Faraday rotation is measured through its influence on the linear polarisation vector as a function of wavelength-squared. The observed Faraday rotation measure, $\mathrm{RM}\left[\mathrm{rad} \mathrm{m}^{-2}\right]$, depends on the line-of-sight magnetic field, $B_{\|}[\mu \mathrm{G}]$, threading a region of ionised gas with electron density, $n_{\mathrm{e}}\left[\mathrm{cm}^{-3}\right]$, along a path length, $l[\mathrm{pc}]$, following

$\mathrm{RM}=0.812 \int_{\text {source }}^{\text {telescope }} n_{\mathrm{e}} B_{\|} \mathrm{d} l$.

In this paper, we present an analysis of the linear polarisation and Faraday rotation properties of an FRII radio galaxy $(\mathrm{J} 1235+5317)$ with a linear size of $3.4 \mathrm{Mpc}$. The observations were done with the LOw Frequency Array (LOFAR; van Haarlem et al. 2013) which provides excellent sensitivity to diffuse extended structures due to the presence of numerous short baselines and exceptional Faraday rotation measure (RM) accuracy, which depends on the total coverage in wavelength-squared. While low frequency radio telescopes provide the best RM accuracy, sources at these frequencies are most strongly affected by Faraday depolarisation (e.g. Burn 1966), which decreases the degree of linear polarisation below the detection limit for many sources (Farnsworth et al. 2011). Despite this there is a growing number of polarised sources being found at low frequencies (e.g. Bernardi et al. 2013; Mulcahy et al. 2014; Jelić et al. 2015; Orrù et al. 2015; Lenc et al. 2016; Van Eck et al. 2018; O'Sullivan et al. 2018; Neld et al. 2018; Riseley et al. 2018).

$\mathrm{J} 1235+5317$ was discovered to be polarised at $144 \mathrm{MHz}$ by Van Eck et al. (2018), in LOFAR data imaged at an angular resolution of $4.3^{\prime}$. The source was first reported by Schoenmakers et al. (2001), and the first optical identification (SDSS J123458.46+531851.3) was proposed by Banfield et al. (2015). However, our new observations show that the previously assumed host galaxy is accidentally located close to the geometric centre between the two lobes and that the real host galaxy is actually connected to the south east (SE) lobe by a faint jet. The radio core is coincident with the galaxy SDSS J123501.52+531755.0, which is identified as PSO J123501.519+531754.911 (Flewelling et al. 2016) for the radio source ILT J123459.82+531851.0 in Williams et al. (2019). Estimates of the photometric redshift of this galaxy are 0.349 (Bilicki et al. 2016), 0.41 (Beck et al. 2016) and 0.44 (Brescia et al. 2014; Duncan et al. 2019).

The host galaxy is identified in Hao et al. (2010) as a red-sequence galaxy and a cluster candidate, GMBCG J188.75636+53.29864. This is intriguing as GRGs are often thought to evolve in underdense galaxy environments (e.g. Mack et al. 1998), however, recent work indicates that they are most likely the oldest sources in the general population of powerful radio galaxies (Hardcastle et al. 2019). In addition, Hao et al. (2010) estimate a total of $\sim 9$ galaxies within $0.5 \mathrm{Mpc}$ with luminosities $L>0.4 L^{*}$, using a weak-lensing scaling relation, which suggests a poor cluster environment. There is also no evidence for a massive cluster at this location in the sky in the Planck thermal Sunyaev-Zeldovich map (Planck Collaboration XXII 2016).

This paper presents a follow-up study using the same LOFAR data as Van Eck et al. (2018), but imaging at higher angular resolution. We also confirm the new optical host identification and determine its spectroscopic redshift as $z \sim 0.34$, giving the projected linear size of 3.4 Mpc. In Sect. 2, we describe the radio polarisation and optical spectroscopic observations. Section 3 presents the physical properties of $\mathrm{J} 1235+5317$, the inference on the properties of its environment based on dynamical modelling of the jets, and the RM and depolarisation behaviour. In Sect. 4 we discuss the results in the context of the study of the intergalactic medium and its magnetisation. The conclusions are listed in Sect. 5. Throughout this paper, we assume a $\Lambda$ CDM cosmology with $\mathrm{H}_{0}=67.8 \mathrm{~km} \mathrm{~s}^{-1} \mathrm{Mpc}^{-1}, \Omega_{M}=0.308$ and $\Omega_{\Lambda}=0.692$ (Planck Collaboration XIII 2016). At the redshift of the source, $1^{\prime \prime}$ corresponds to a linear size of $5.04 \mathrm{kpc}$. We define the total intensity spectral index, $\alpha$, such that the observed total intensity (I) at frequency $v$ follows the relation $I_{v} \propto v^{+\alpha}$.

\section{Observations and data analysis}

\subsection{Radio observations}

The target source $\mathrm{J} 1235+5317$ was observed as part of the LOFAR Two-Metre Sky Survey (LoTSS; Shimwell et al. 2017, 2019), which is observing the whole northern sky with the LOFAR High-Band Antenna (HBA) from 120 to $168 \mathrm{MHz}$. The data relevant to our target were observed in full polarisation for $8 \mathrm{~h}$ on 26 June 2014, as part of the observing program LC2_038 and with a pointing centre of $\mathrm{J} 200012^{\mathrm{h}} 38^{\mathrm{m}} 06^{\mathrm{s}} .7,+52^{\circ} 07^{\prime} 19^{\prime \prime}$. This gives a distance of $\sim 1.26^{\circ}$ of the target $\mathrm{J} 1235+5317$ from the pointing centre (the FWHM of the primary beam is $\sim 4^{\circ}$ ). Direction-independent calibration was performed using the PREFACTOR pipeline ${ }^{1}$, as described in detail in Shimwell et al. (2017) and de Gasperin et al. (2018), which includes the ionospheric RM correction using RMEXTRACT ${ }^{2}$. Residual ionospheric RM correction errors of $\sim 0.05 \mathrm{rad} \mathrm{m}^{-2}$ are estimated between observations (Van Eck et al. 2018), while slightly larger errors of $\sim 0.1-0.3 \mathrm{rad} \mathrm{m}^{-2}$ are estimated across a single $8 \mathrm{~h}$ observation (Sotomayor-Beltran et al. 2013).

The resulting measurement set, after the PREFACTOR pipeline, has a time resolution of $8 \mathrm{~s}$ and a frequency resolution of $97.6 \mathrm{kHz}$. The direction-independent calibrated data are used throughout for the polarisation and rotation measure analysis, while the direction-dependent calibrated total intensity image (Shimwell et al. 2019) is used to determine the source morphological properties with high precision and for the identification of the host galaxy location. Analysis of polarisation and rotation measure data products after direction-dependent calibration will be presented in future work.

\subsection{Polarisation and Faraday rotation imaging}

To analyse the polarisation and Faraday rotation properties of the target, we phase-shifted the calibrated uv-data to the coordinates of the host galaxy $\left(12^{\mathrm{h}} 35^{\mathrm{m}} 01^{\mathrm{s}} .5,+53^{\circ} 17^{\prime} 55^{\prime \prime}\right)$, which lies almost at the centre of the extended emission. We calibrated the data for short-timescale phase variations caused by the ionosphere, then averaged to $32 \mathrm{~s}$ to reduce the data size and to help speed up the subsequent imaging, while avoiding

$\begin{array}{ll}1 & \text { https://github.com/lofar-astron/prefactor } \\ 2 & \text { https://github.com/lofar-astron/RMextract }\end{array}$ 
any significant time smearing (e.g. Neld et al. 2018). Both the phase-shifting and time-averaging were done using NDPPP (van Diepen \& Dijkema 2011) $)^{3}$. The imaging software wSCLEAN (Offringa \& McKinley 2014) ${ }^{4}$ was used to create $I, Q, U, V$ channel images at $97.6 \mathrm{kHz}$ resolution, for a $25^{\prime}$ field of view ( $\sim$ twice the linear size of $\mathrm{J} 1235+5317$ ). A minimum uv-range of $150 \lambda$ was used to avoid sensitivity to Galactic polarised emission on scales of $\gtrsim 25^{\prime}$. The maximum uv-range was set to $18 \mathrm{k} \lambda$, and combined with a Briggs weighting of 0 , resulted in a beam size of $26^{\prime \prime} \times 18^{\prime \prime}$, sampled with $3^{\prime \prime} \times 3^{\prime \prime}$ pixels. The differential beam correction per channel was applied using WSCLEAN, as the correction for the LOFAR beam gain at the pointing centre was already applied during the initial calibration of the data. All channel images with $Q$ or $U$ noise higher than five times the average noise level were removed from subsequent analysis, leaving a total of 404 images covering $120-167 \mathrm{MHz}$ (with a central frequency of $143.5 \mathrm{MHz}$ ).

RM synthesis and RMCLEAN (Brentjens \& de Bruyn 2005; Heald et al. 2009) were then applied to the $Q$ and $U$ images using PYRMSYNTH $^{5}$. The data have an RM resolution of $1.16 \mathrm{rad} \mathrm{m}^{-2}$, are sensitive to polarised emission from Faraday thick regions up to $\sim 0.98 \mathrm{rad} \mathrm{m}^{-2}$, and $|\mathrm{RM}|$ values for Faraday thin regions as high as $450 \mathrm{rad} \mathrm{m}^{-2}$ can be detected. An RM cube with a Faraday depth $(\phi)$ axis covering $\pm 500 \mathrm{rad} \mathrm{m}^{-2}$ and sampled at $0.5 \mathrm{rad} \mathrm{m}^{-2}$ intervals was constructed for initial inspection of the data. The concept of Faraday depth (Burn 1966) can be useful to introduce here to describe regions with complicated distributions of Faraday rotation along the line of sight, such as multiple distinct regions of polarised emission experiencing different amounts of Faraday rotation, which could be identified through multiple peaks in a Faraday depth spectrum or Faraday dispersion function (FDF). As no significant emission was found at large Faraday depths, the final RM and polarisation images were constructed from FDFs with a range of $\pm 150 \mathrm{rad} \mathrm{m}^{-2}$, sampled at $0.15 \mathrm{rad} \mathrm{m}^{-2}$. To identify peaks in the FDF, a threshold of $8 \sigma_{Q U}$ was used, where $\sigma_{Q U}$ is calculated from the outer $20 \%$ of the Faraday depth range in the RMCLEAN $Q$ and $U$ spectra. The mean $\sigma_{Q U}$ across the field was $\sim 90 \mu \mathrm{Jy}_{\text {beam }}{ }^{-1}$. Since no correction was made for the instrumental polarisation, peaks in the Faraday dispersion function appears near $\phi \sim 0 \mathrm{rad} \mathrm{m}^{-2}$ at a typical level of $\sim 1.5 \%$ of the Stokes $I$ emission. This instrumental polarisation signal is also smeared out by the ionospheric RM correction making it difficult to identify real polarised emission at low Faraday depths $\left(\lesssim \pm 3 \mathrm{rad} \mathrm{m}^{-2}\right)$. Thus, when identifying real polarised emission peaks in the FDF, the range $\pm 3 \mathrm{rad} \mathrm{m}^{-2}$ is excluded. RM and polarised intensity images are created from the brightest, real polarised peak above $8 \sigma_{Q U}$ at each pixel, after fitting a parabola around the peak to obtain the best-fitting RM and polarised intensity. In the case of the polarised intensity image, a correction for the polarisation bias was also made following George et al. (2012). The error in the RM at each pixel was calculated in the standard way as the RM resolution divided by twice the signal to noise ratio of the detection (Brentjens \& de Bruyn 2005).

A full-band Stokes $I$ image was made using the same image parameters as the channel images specified above, with multiscale cleaning applied for an automatic threshold of $3 \sigma$ and deeper cleaning (to $0.3 \sigma$ ) within an automatic masked region created from the clean components. The degree-of-polarisation image was created by dividing the band-averaged polarised

\footnotetext{
https://support . astron. nl/LOFARImagingCookbook/

4 https://sourceforge.net/projects/wsclean

5 https://github.com/mrbell/pyrmsynth
}

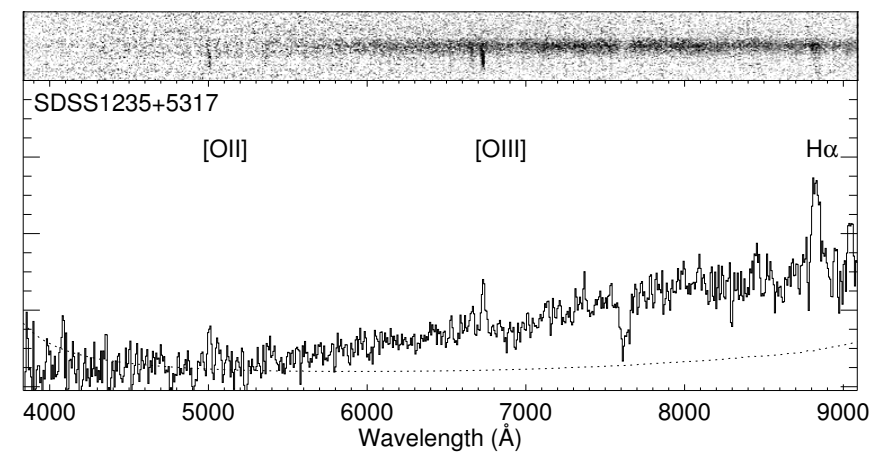

Fig. 1. Optical spectrum of the host galaxy SDSS J123501.52+ 531755.0 taken with AIFOSC instrument on the Nordic Optical Telescope, which shows emission lines $\mathrm{H} \alpha$, [OII] and [OIII] at a redshift of 0.34 .

intensity image from RM synthesis (with a cutoff at $8 \sigma_{Q U}$ ) by the full-band Stokes $I$ image (with a cutoff at 3 times the local noise level).

\subsection{Optical spectroscopic observations}

SDSS J123501.52+531755.0 was observed with the Nordic Optical Telescope on March 25 and March 262018 for a total integration time of $5400 \mathrm{~s}$. We used the Andalucia Faint Object Spectrograph and Camera (AlFOSC) and a 1.3 arcsec wide longslit and grism 4 with 300 rules per millimetre providing a spectral resolution of 280 and a useful spectral range of 3800 $9100 \AA$. The slit was placed at a parallactic angle of $60^{\circ}$ east of north on both nights at the onset of integration. The airmass ranged from 1.20 to 1.15 . The observing conditions were poor with a variable seeing above 2 arcsec and with passing clouds. Despite this we clearly detected several emission lines (Fig. 1) consistent with a mean redshift of $0.3448 \pm 0.0003$ (1-sigma error). The [OII] and [OIII] images have a peculiar morphology extending away from the continuum source to the northern side of the galaxy. In particular [OIII], $\lambda 5008 \AA$ can be traced over 4 arcsec below the continuum trace $(20 \mathrm{kpc}$ at $z=0.34)$. This indicates the presence of an extended emission line region.

\section{Results}

\subsection{Radio morphology of J1235+5317}

Figure 2 shows the total intensity image at 6 " resolution from the LoTSS direction-dependent calibrated data (Shimwell et al. 2019). This provides the best radio image to date for this source, enabling an unambiguous host galaxy identification with SDSS J123501.52+531755.0. The noise level in the image ranges from $\sim 70 \mu \mathrm{Jy}$ beam $^{-1}$ in areas away from bright sources to $\sim 100 \mu \mathrm{Jy} \mathrm{beam}^{-1}$ near the hotspots/lobes.

The core of this FRII radio galaxy, located at J2000 $12^{\mathrm{h}} 35^{\mathrm{m}} 01^{\mathrm{s}} .5,+53^{\circ} 17^{\prime} 55^{\prime \prime}$, has an integrated flux density of $\sim 1.1 \mathrm{mJy}$ at $144 \mathrm{MHz}$ and $1.4 \mathrm{GHz}$ (FIRST; Becker et al. 1995) suggesting a flat spectrum. However, the core is also detected in the $\operatorname{VLASS}^{6}$ Quick-Look (QL) image at $3 \mathrm{GHz}(\sim 2.9 \mathrm{mJy})$ and the 9C catalogue (Waldram et al. 2010) at $15 \mathrm{GHz}(\sim 4 \mathrm{mJy})$ indicating an inverted spectral index of $\alpha_{\text {core }} \sim+0.3$ when combined with the LoTSS core flux density. As the LoTSS, VLASS and 9C observations are closest in time, we consider the core to have

\footnotetext{
6 https://archive-new . nrao.edu/vlass/
} 


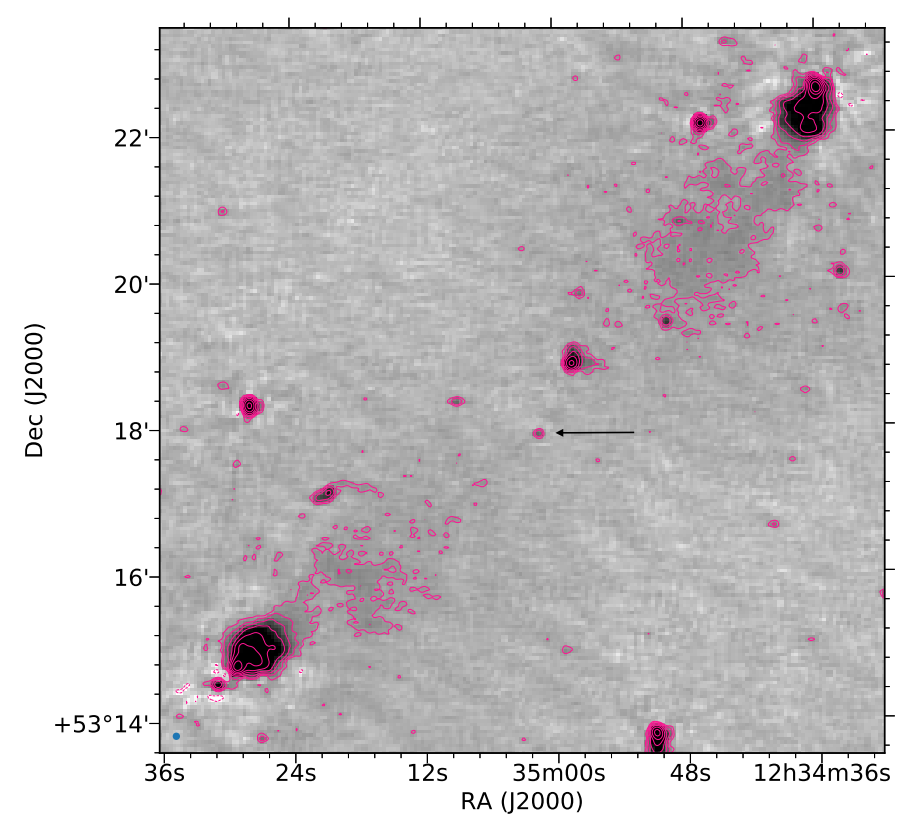

Fig. 2. LoTSS total intensity image at $144 \mathrm{MHz}$ at $6^{\prime \prime}$ resolution (after direction-dependent calibration). The contours start at $300 \mu \mathrm{Jy} \mathrm{beam}^{-1}$ and increase by factors of 2 (with one negative contour at $-300 \mu \mathrm{Jy} \mathrm{beam}^{-1}$ ). The greyscale image is tuned to show the noise variation across the image $\left(\sim 70 \mu \mathrm{Jy}\right.$ beam $^{-1}$ away from bright sources and $\sim 100 \mu \mathrm{Jy}_{\text {beam }}{ }^{-1}$ near the hotspots), as well as a faint hint of the south-east jet. The radio galaxy core coincident with the host galaxy SDSS J123501.52+531755.0 is indicated by the horizontal arrow. The synthesised beam size is shown in the bottom left hand corner of image.

an inverted spectral index, with time variability explaining the lower than expected flux density from FIRST at $1.4 \mathrm{GHz}$. There is also a faint hint of a jet connecting the host with the south-east (SE) lobe. If this is real, then it suggests that the SE jet and lobe are orientated slightly towards us on the sky.

Using the $3 \sigma$ contour to define the lobe edges, we find the lobes have a width of $\sim 83^{\prime \prime}$ and $\sim 94^{\prime \prime}$, giving an axial ratio of $\sim 4.4$ for the north-west (NW) lobe and $\sim 3.3$ for the SE lobe, respectively. This is consistent with the typical axial ratios from 2 to 7 for the lobes of most (smaller) GRGs (e.g. Machalski et al. 2006). In Table 1, we compile the integrated flux densities of the NW and SE lobes and hotspots from both current and archival data. The integrated flux densities of the NW lobe and hotspot are slightly higher than the SE lobe and hotspot at $144 \mathrm{MHz}$, with both having spectral index values of $\alpha_{\text {lobe }} \sim-0.8$. The NW hotspot is resolved into primary and secondary hotspot regions in the VLASS at $3 \mathrm{GHz}\left(2.4^{\prime \prime} \times 2.1^{\prime \prime}\right.$ beam $)$, while the SE hotspot maintains a single component.

The straight-line distance from the core to the NW hotspot is $\sim 365^{\prime \prime}$ (1.84 Mpc), compared to $\sim 311^{\prime \prime}(1.56 \mathrm{Mpc})$ from the core to the SE hotspot, giving a lobe length ratio of 1.17. The inferred jet-misalignment (from co-linearity) of $\sim 13.6^{\circ}$ is most likely due to bending of the NW and/or SE jets on large scales, as is sometimes observed in other FRII radio sources (Black et al. 1992). We expect that the lobe-length asymmetry and jet-misalignment are caused by interactions between the jet and the external environment on large scales, as opposed to light travel time effects (Longair \& Riley 1979). Asymmetries in the jet and lobe lengths of GRGs are often attributed to interactions with the large scale structure environment (Pirya et al. 2012; Malarecki et al. 2015). The advancing NW jet may be influenced by a nearby filament (see Sect. 4.4.1 and the filament in the $z \sim 0.335$ slice), although deeper optical spectroscopic observations would be required to determine whether or not this filament is indeed close enough in redshift to that of the host galaxy to have an influence.

\subsection{Faraday rotation measure distribution}

Figure 3 shows the RM distribution for J1235+5317, using an $8 \sigma_{Q U}$ threshold, overlaid by Stokes $I$ contours at the same angular resolution. The Faraday dispersion functions for the brightest pixel in polarised intensity in each lobe are also shown, with a red cross marking the peak polarisation at which the RM was found. Other peaks in the spectrum are either noise peaks or related to the instrumental polarisation near $\mathrm{RM} \sim 0 \mathrm{rad} \mathrm{m}^{-2}$. The RM distributions of each lobe are shown in Fig. 4. The mean and standard deviation of the RM are $+7.42 \mathrm{rad} \mathrm{m}^{-2}$ and $0.07 \mathrm{rad} \mathrm{m}^{-2}$ for the NW lobe, and $+9.92 \mathrm{rad} \mathrm{m}^{-2}$ and $0.11 \mathrm{rad} \mathrm{m}^{-2}$ for the SE lobe, respectively. The median RM errors for the NW and SE lobe regions are $0.04 \mathrm{rad} \mathrm{m}^{-2}$ and $0.06 \mathrm{rad} \mathrm{m}^{-2}$. The mean $\mathrm{RM}$ difference between the lobes of $2.5 \pm 0.1 \mathrm{rad} \mathrm{m}^{-2}$ is thus highly significant. At the angular separation of the lobes $\left(11^{\prime}\right)$, systematic errors in the ionospheric RM correction would affect both lobes equally and thus do not contribute to the RM difference between the lobes. We can estimate the significance of the small RM variations within each lobe accounting for the number of pixels in each synthesised beam following Leahy et al. (1986), where a reduced-chi-squared of $\sim 1$ is expected if noise errors dominate the RM fluctuations. We find no evidence for the detection of significant RM variations across the NW lobe, with a reduced-chi-squared of 1.1. However, a reduced-chi-squared of 1.8 provides evidence, at a level of $\sim 1.35 \sigma$, for RM variations across the SE lobe of $\sim 0.1 \mathrm{rad} \mathrm{m}^{-2}$.

\subsection{Faraday depolarisation}

The polarised intensity and degree of polarisation distributions are shown in Fig. 3. The NW lobe is much brighter with a peak polarised intensity of $6.5 \mathrm{mJy}^{-1}$ beam $^{-1}$ (coincident with the hotspot) and a degree of polarisation of $4.9 \%$ at that location (ranging from $1.2 \%$ to $5.1 \%$ across the detected emission). The SE lobe is fainter with a peak polarised intensity of $1.1 \mathrm{mJy}$ beam $^{-1}$. The degree of polarisation at that location is $2.8 \%$, and it ranges from 1.1 to $3.3 \%$ across the lobe. The nondetection of polarised emission from the SE hotspot is likely due to intrinsic non-uniform field structures and Faraday depolarisation on scales smaller than the resolution of our observations. The fainter, extended lobe emission would have to be $\gtrsim 10 \%$ polarised to be detected in these observations.

In order to estimate the amount of depolarisation between $1.4 \mathrm{GHz}$ and $144 \mathrm{MHz}$, the LoTSS data were compared with those of the NRAO VLA Sky Survey (NVSS; Condon et al. 1998). To determine the degree of polarisation at the same angular resolution as the NVSS survey, the RM pipeline was reapplied to the LoTSS data imaged at a lower angular resolution of $\sim 45^{\prime \prime}$.

At the peak polarised intensity location in the NW lobe of the LOFAR image, matched to the NVSS resolution, the degree of polarisation is $4.0 \pm 0.3 \%$. At the same location in the NVSS image at $1.4 \mathrm{GHz}$, the degree of polarisation is $6.4 \pm 1.4 \%$. This gives a depolarisation factor of $\mathrm{DP}_{1400}^{144} \sim 0.6$, where $\mathrm{DP}_{1400}^{144}$ is the degree of polarisation at $144 \mathrm{MHz}$ divided by the degree of polarisation at $1.4 \mathrm{GHz}$. Assuming the commonly used external Faraday dispersion model for depolarisation, $p(\lambda) \propto \mathrm{e}^{-2 \sigma_{\mathrm{RM}}^{2} \lambda^{4}}$ (Burn 1966), provides a value of $\sigma_{\mathrm{RM}} \sim 0.1 \mathrm{rad} \mathrm{m}^{-2}$. 

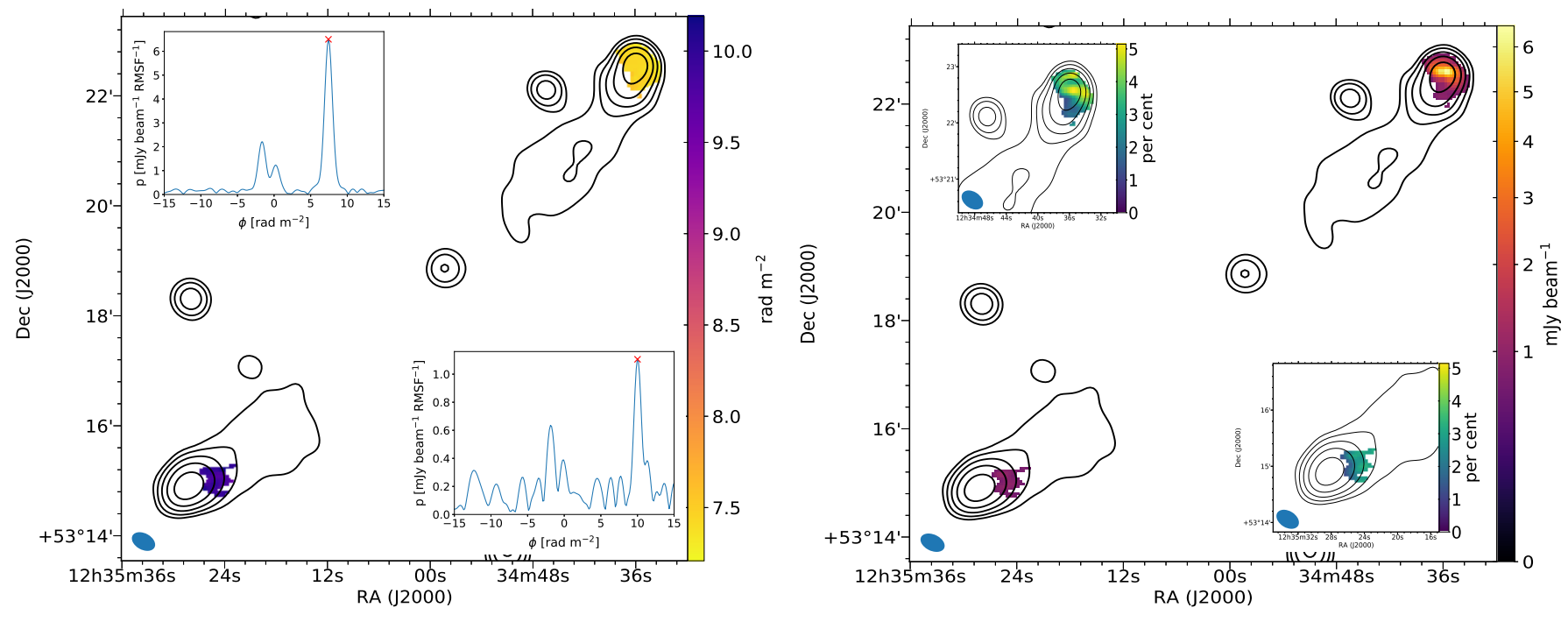

Fig. 3. Left image: main image: Faraday rotation measure distribution (colour scale) of the north-west (NW) and south-east (SE) lobe regions that are detected above the threshold of $8 \sigma_{Q U}$, overlaid by the total intensity contours starting at $5 \mathrm{mJy}$ beam ${ }^{-1}$ and increasing in factors of two. Insets: The absolute value of the RMCLEAN Faraday dispersion function for the brightest polarised pixel in the NW lobe (top) and SE lobe (bottom). Right image: main image: polarised intensity greyscale, in $\mathrm{mJy} \mathrm{beam}^{-1}$, overlaid by the total intensity contours. Insets: degree of polarisation colourscale (in per cent) from zoomed in regions of the NW and SE lobes.
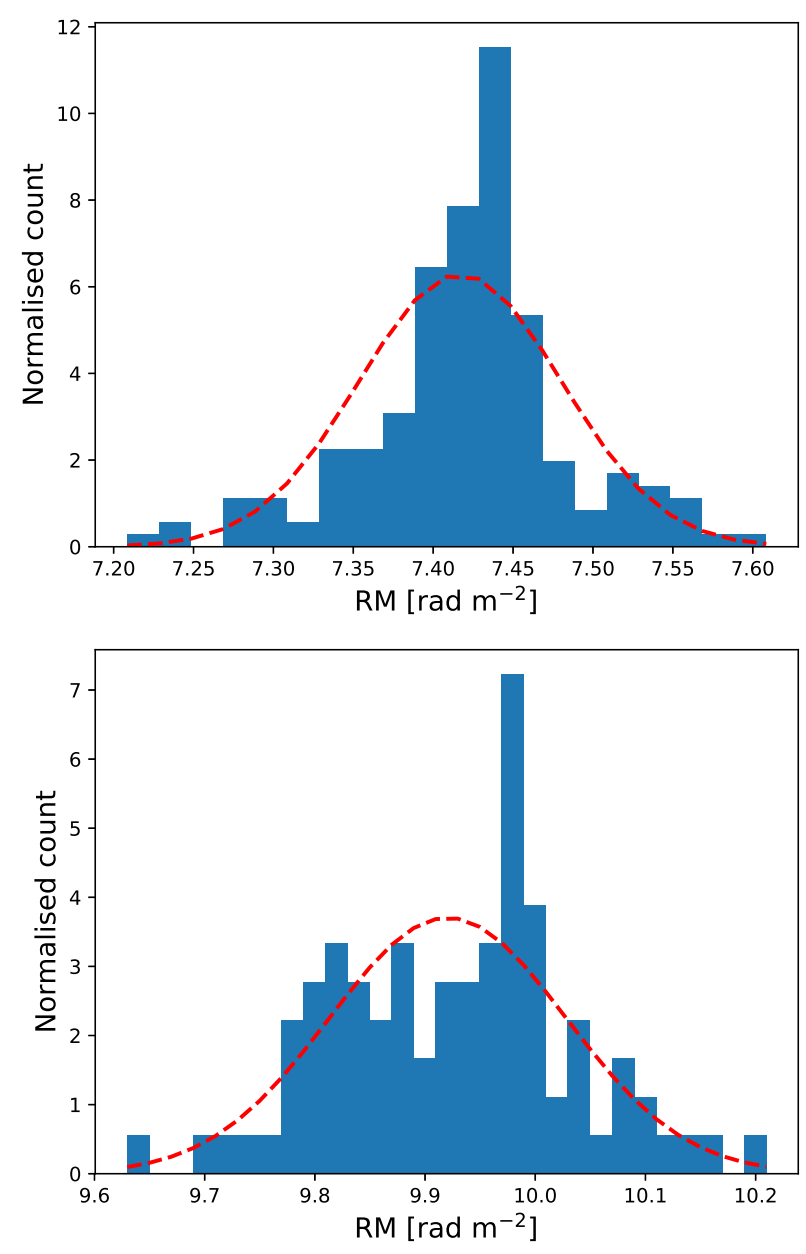

Fig. 4. Histograms of the RM distribution from the north-west lobe (top panel) and south-east lobe (bottom panel) regions of $\mathrm{J} 1235+5317$. The red dashed line shows a Gaussian distribution with the same mean and standard deviation as the observed data.
For the SE lobe, the degree of polarisation at the peak polarised intensity at $144 \mathrm{MHz}$ is $1.8 \pm 0.7 \%$ (at $45^{\prime \prime}$ resolution) and $10.1 \pm 2.1 \%$ at the same location at $1.4 \mathrm{GHz}$. This gives $\mathrm{DP}_{1400}^{144} \sim 0.2$, corresponding to larger amounts of depolarisation than in the NW lobe. In the case of external Faraday dispersion, this corresponds to $\sigma_{\mathrm{RM}} \sim 0.2 \mathrm{rad} \mathrm{m}^{-2}$.

The observed difference in depolarisation between the NW and SE lobes may be due to the different location within each lobe from which the polarised emission arises. In the case of the NW lobe, the peak polarised emission is coincident with the hotspot location, whereas in the SE lobe, the peak polarised emission is significantly offset from the hotspot $\left(\sim 40^{\prime \prime}\right.$ away, in the bridge emission, with the offset also present in the NVSS images). Furthermore, from the non-detection of polarisation in the SE hotspot at $144 \mathrm{MHz}$, with a degree of polarisation $<0.35 \%$, we can place a lower limit on the Faraday depolarisation at this location of $\sigma_{\mathrm{RM}} \sim 0.25 \mathrm{rad} \mathrm{m}^{-2}$, based on comparison with the NVSS degree of polarisation of $\sim 5 \%$ at this location.

From inspection of the VLASS QL image at $3 \mathrm{GHz}$, the physical extent of the NW hotspot $\left(\sim 2.4^{\prime \prime}\right)$ is smaller compared to the SE lobe region (of order $20^{\prime \prime}$ in size) and thus less affected by depolarisation caused by RM variations within the synthesised beam at $144 \mathrm{MHz}$. Since the amount of depolarisation scales roughly as the square-root of the number of Faraday rotation cells, this could reasonably explain the difference in the observed depolarisation between the lobes. However, the enhanced depolarisation at the location of the SE hotspot is more difficult to explain and may indicate a significant interaction between the hotspot/lobe magnetic field and the ambient medium. This warrants further investigation with more sensitive observations at low frequencies.

Overall, given the small amount of observed Faraday depolarisation, it is important to consider the accuracy of the correction for Faraday rotation from the ionosphere. Van Eck et al. (2018) estimate a residual error in the ionosphere RM correction between observations of $0.05 \mathrm{rad} \mathrm{m}^{-2}$. As the ionosphere $\mathrm{RM}$ corrections across an observation (i.e. $8 \mathrm{~h}$ ) are linearly interpolated in time between direct estimates every $2 \mathrm{~h}$, a rough 
Table 1. Archival and measured flux densities, as well as the best-fit flux densities (in the self-consistent, s.c., fits) for the north-west and south-east lobes of $\mathrm{J} 1235+5317$.

\begin{tabular}{|c|c|c|c|c|c|c|}
\hline \multirow[b]{2}{*}{$\begin{array}{l}\text { Freq. } \\
\text { (MHz) } \\
(1) \\
\end{array}$} & \multicolumn{3}{|c|}{$N$-lobe } & \multicolumn{3}{|c|}{$S$-lobe } \\
\hline & $\begin{array}{r}\text { Entire Lobe } \\
(\mathrm{mJy}) \\
(2) \\
\end{array}$ & $\begin{array}{c}\text { Hotspots } \\
\text { (mJy) } \\
(3)\end{array}$ & $\begin{array}{l}\text { s.c. fit } \\
(\mathrm{mJy}) \\
(4)\end{array}$ & $\begin{array}{r}\text { Entire Lobe } \\
(\mathrm{mJy}) \\
(5) \\
\end{array}$ & $\begin{array}{c}\text { Hotspots } \\
(\mathrm{mJy}) \\
(6)\end{array}$ & $\begin{array}{l}\text { s.c. fit } \\
\text { (mJy) } \\
(7)\end{array}$ \\
\hline $143.6^{i}$ & $403 \pm 40$ & $151 \pm 21$ & 356.6 & $378 \pm 40$ & $132 \pm 25$ & 345.3 \\
\hline $151^{a}$ & $350 \pm 52$ & & 344.4 & $320 \pm 52$ & & 333.3 \\
\hline $151^{b}$ & $375 \pm 32$ & & 344.4 & $302 \pm 31$ & & 333.3 \\
\hline $325^{c}$ & $177 \pm 36$ & & 193.0 & $149 \pm 36$ & & 185.1 \\
\hline $325^{i}$ & $154 \pm 58$ & & 193.0 & $153 \pm 58$ & & 185.1 \\
\hline $408^{d}$ & $160 \pm 40$ & & 160.6 & $145 \pm 34$ & & 153.2 \\
\hline $1400^{e}$ & $59 \pm 4$ & & 55.9 & $50 \pm 2$ & & 51.0 \\
\hline $1400^{i}$ & $55 \pm 19$ & $36 \pm 4$ & 55.9 & $47 \pm 19$ & $33 \pm 5$ & 51.0 \\
\hline $2980^{g}$ & & $21 \pm 3$ & & & $20 \pm 3$ & \\
\hline $4850^{f}$ & $21 \pm 4$ & & 18.2 & $18.4 \pm 4$ & & 15.6 \\
\hline $15200^{h}$ & $(5.2 \pm 2)$ & $5.2 \pm 1$ & 6.3 & $(6.6 \pm 2)$ & $6.6 \pm 1$ & 5.1 \\
\hline
\end{tabular}

References. ${ }^{(a)} 6 \mathrm{C} 3$ (Hales et al. 1990); ${ }^{(b)} 7 \mathrm{Cn}$ (Riley et al. 1999); ${ }^{(c)}$ WENSS (Rengelink et al. 1997); ${ }^{(d)}$ B3.3 (Pedani \& Grueff 1999); ${ }^{(e)}$ NVSS (Condon et al. 1998); ${ }^{(f)}$ GB6 (Gregory et al. 1996); ${ }^{\left({ }^{g}\right)}$ VLASS (Lacy et al. in prep.); ${ }^{(h)} 9 \mathrm{Cc}$ (Waldram et al. 2010); ${ }^{(i)}$ this paper.

estimate can be made for the residual error within the observation of $\sim 0.05 \sqrt{4} \sim 0.1 \mathrm{rad} \mathrm{m}^{-2}$. This means that most (or all) of the observed depolarisation in the NW hotspot is possibly due to residual errors in the ionospheric RM correction. However, the difference in depolarisation between the NW hotspot and SE lobe cannot be explained by ionosphere RM errors. Therefore, a $\sigma_{\mathrm{RM}}$ of at least $\sim 0.1 \mathrm{rad} \mathrm{m}^{-2}$ in the SE lobe can be considered astrophysically meaningful. This is comparable to the RM variations across the SE lobe of $\sim 0.1 \mathrm{rad} \mathrm{m}^{-2}$ found in Sect. 3.2.

\subsection{Dynamical modelling}

In order to decouple the properties of the electron density and magnetic field along the line of sight in the measured Faraday rotation and depolarisation, additional information is required on the physical characteristics of $\mathrm{J} 1235+5317$ (i.e. the magnetic field strength of the emission region) and the properties of its surrounding environment (i.e. the ambient gas density). These properties can be estimated through dynamical modelling of the radio lobes, while simultaneously accounting for energy losses of relativistic particles (electrons and positrons) injected into the expanding lobes by the relativistic jets (e.g. Machalski et al. 2011, 2016, and references therein). This is important because we lack X-ray data that could constrain the properties of the external medium (e.g. Ineson et al. 2017) and/or the magnetic field strength of the hotspot and lobes, without the need for the assumption of equipartition between the radiating particles and magnetic field (e.g. Mingo et al. 2017). Therefore, here we apply the evolutionary DYNAGE code of Machalski et al. (2007) to the radio lobes of $\mathrm{J} 1235+5317$, primarily to obtain an estimate of the external gas density, as well as estimates for the magnetic field strength of the lobes. The fitting procedure is performed separately for each lobe using the observational data given in Sect. 3.1, together with the radio luminosities calculated from the flux densities listed in Table 1. The input model parameters that are assumed are given in Table 2.

Characteristic of almost all FRII sources is a modest asymmetry in the length and radio luminosity of the lobes. Therefore, as might be expected, the DYNAGE results for the jet power $Q_{\mathrm{j}}$,
Table 2. Dynamical modelling input model parameters.

\begin{tabular}{|c|c|c|}
\hline $\begin{array}{l}\text { Parameter } \\
\text { (1) }\end{array}$ & $\begin{array}{c}\text { Symbol } \\
(2)\end{array}$ & $\begin{array}{c}\text { Value } \\
(3)\end{array}$ \\
\hline Set: & & \\
\hline Adiabatic index of the lobes' material & $\Gamma_{\mathrm{lb}}$ & $4 / 3$ \\
\hline Adiabatic index of the ambient medium & $\Gamma_{\mathrm{x}}$ & $5 / 3$ \\
\hline Adiabatic index of the lobes' magnetic field & $\Gamma_{\mathrm{B}}$ & $4 / 3$ \\
\hline Minimum electron Lorentz factor (injected) & $\gamma_{\min }$ & 1 \\
\hline Maximum electron Lorentz factor (injected) & $\gamma_{\max }$ & $10^{7}$ \\
\hline $\begin{array}{l}\text { Core radius of power-law } \\
\text { ambient density distribution }\end{array}$ & $a_{0}$ & $10 \mathrm{kpc}$ \\
\hline $\begin{array}{l}\text { Initial slope of power-law } \\
\text { ambient density distribution }\end{array}$ & $\beta$ & 1.5 \\
\hline Thermal particles within the lobes & $k$ & 0 \\
\hline $\begin{array}{l}\text { Jet viewing angle } \\
\text { Free: }\end{array}$ & $\theta$ & $90^{\circ}$ \\
\hline Jet power & $Q_{\mathrm{j}}\left(\mathrm{erg} \mathrm{s}^{-1}\right)$ & \\
\hline External density at core radius & $\rho_{0}\left(\mathrm{~g} \mathrm{~cm}^{-3}\right)$ & \\
\hline $\begin{array}{l}\text { Exponent of initial power-law energy } \\
\text { distribution of relativistic particles } \\
\text { Source (lobe) age }\end{array}$ & $\begin{array}{c}p=1+2 \alpha_{\text {inj }} \\
t(\mathrm{Myr})\end{array}$ & \\
\hline
\end{tabular}

the central density of the external medium $\rho_{0}$, and other physical parameters can appear different for the two lobes of the same source. This aspect has been analysed by Machalski et al. (2009, 2011) for a sample of thirty GRGs. While some of the differences were within the uncertainties of the fitted values for the model parameters, significant differences were possible in cases where the evolution of the magnetic field and/or various energy losses and acceleration processes of the relativistic particles are different at the hotspots of the opposite lobes. Alternatively, such differences, especially in GRGs, may reflect different external conditions well beyond the host galaxy and cluster/group environment.

Following Machalski et al. (2009), we averaged the values of $Q_{\mathrm{j}}$ and $\rho_{0}$ initially found in the "independent solution" and treated them as fixed parameters in the "self-consistent" model, $\left\langle Q_{\mathrm{j}}\right\rangle$ and $\left\langle\rho_{0}\right\rangle$, respectively. New values of the slope of the ambient density distribution $(\beta)$ and the age $(t)$ for the NW and SE lobes, are denoted as $\beta_{\text {s.c. }}$ and $t_{\text {s.c. }}$ (Table 3 ). The DYNAGE fits to the observed data points are shown with solid lines in Fig. 5. Table 3 presents the derived physical properties of the lobes, including a minimum-energy magnetic field strength in the lobes of $B_{\mathrm{me}} \sim 1 \mu \mathrm{G}$ and an external density of $\sim 2 \times$ $10^{-31} \mathrm{~g} \mathrm{~cm}^{-3}$ (i.e. $n_{\mathrm{e}} \sim 10^{-7} \mathrm{~cm}^{-3}$ ). This density is similar to the mean density of the Universe assuming half the baryons are in the WHIM (Machalski et al. 2011), and implies that the radio lobes are likely propagating into a low-density region of the Universe.

We also used the synchrotron minimum energy (equipartition) magnetic field formulation in Worrall et al. (2006) to estimate the lobe magnetic field strength. From this we find an equipartition magnetic field strength that is 2.6 times higher than the $1 \mu \mathrm{G}$ derived from the dynamical modelling (for $\gamma_{\min }=10$ ). When calculated in this manner the lobe equipartition field strength is usually found to be overestimated, by a factor of 2 to 3 , compared to that found from X-ray Inverse Compton observations of lobes (e.g. Ineson et al. 2017; Mingo et al. 2017). This highlights some of the uncertainties in the calculation of equipartition magnetic field strengths in radio galaxies (e.g. Beck \& Krause 2005; Konar et al. 2008). Here we adopt the lobe magnetic field strength obtained from the dynamical 


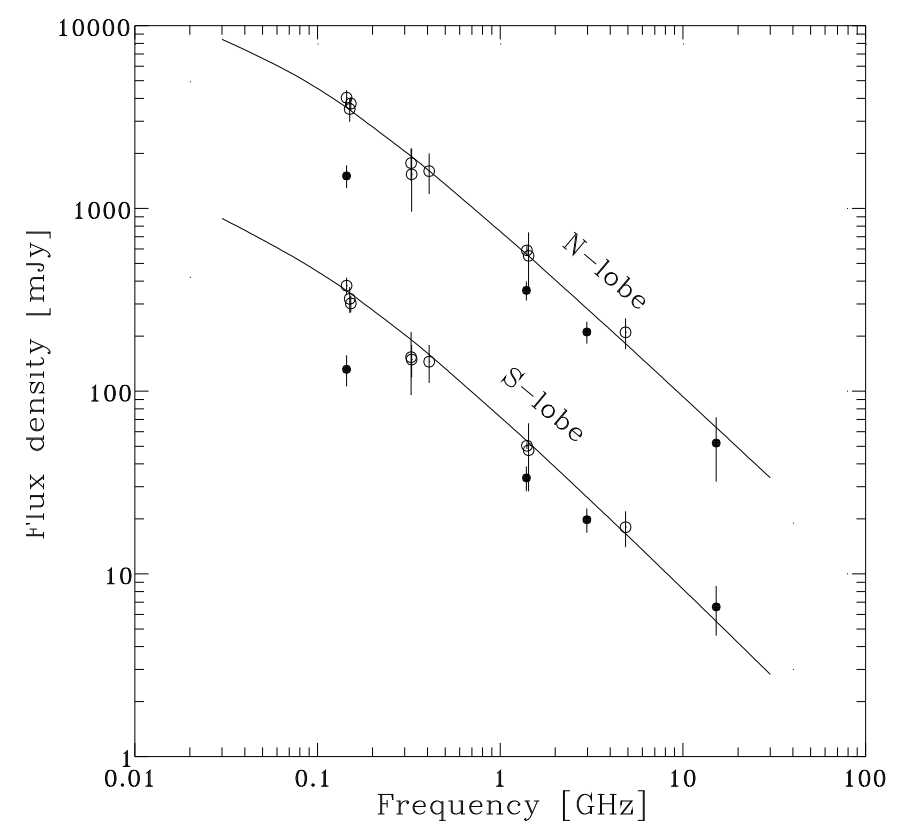

Fig. 5. DYNAGE fits (solid lines) to the total intensity spectra of the north-west and south-east lobes (open circles), and the spectral points of the hotspot regions (filled dots; not used in the fits). Note that the north-west lobe flux density scale is shifted one decade up in relation to the given ordinate scale.

modelling as it takes into account more physical effects, such as the jet power, adiabatic expansion and age of the lobes.

\section{Interpretation}

The difference in the mean RM between the NW and SE lobes is $2.5 \pm 0.1 \mathrm{rad} \mathrm{m}^{-2}$. This may be due to variations in the Galactic RM (GRM) on scales of $\sim 11^{\prime}$, differences in the magnetoionic material of the intergalactic medium on large scales, and/or line-of-sight path length differences towards either lobe. The observed Faraday depolarisation of $\sigma_{\mathrm{RM}} \sim 0.1 \mathrm{rad} \mathrm{m}^{-2}$ associated with the SE lobe could be due to small scale fluctuations of the magnetic field in the local external medium and/or from Faraday rotation internal to the source. Constraining the likelihood of these possibilities requires some considerations of the expected variations in the GRM, knowledge of the geometry and physical properties of the radio lobes, and details of the environment surrounding the radio galaxy and in the foreground.

\subsection{Galactic $R M$ variations}

The reconstruction of the GRM by Oppermann et al. (2012, 2015) gives $+14.8 \pm 4.5 \mathrm{rad} \mathrm{m}^{-2}$ across both the NW and SE lobe (the Galactic coordinates of $\mathrm{J} 1235+5317$ are $l=128.46^{\circ}$, $b=63.65^{\circ}$ ). This is higher than the mean RMs of +7.4 and $+9.9 \mathrm{rad} \mathrm{m}^{-2}$ found for the NW and SE lobes, respectively. However, it should be kept in mind that the LoTSS RM values have been corrected for the time-variable ionosphere RM $(+1.6$ to $+1.9 \mathrm{rad} \mathrm{m}^{-2}$ ), while the catalogue from which the GRM map is mainly made (Taylor et al. 2009) does not have this correction applied. Thus, the RM of the NW and SE lobe are within the 1-sigma and 2-sigma errors in the GRM, respectively.

The variation in the GRM map for three adjacent pixels (in the direction of the largest gradient) across the source is
Table 3. Fitted values of the model free-parameters in the "selfconsistent" dynamical modelling solution.

\begin{tabular}{lccc}
\hline \hline $\begin{array}{l}\text { Parameter } \\
(1)\end{array}$ & $\begin{array}{c}\text { Symbol } \\
(2)\end{array}$ & $\begin{array}{c}\text { Value for } N \text {-lobe } \\
(3)\end{array}$ & $\begin{array}{c}\text { Value for } S \text {-lobe } \\
(4)\end{array}$ \\
\hline Initial effective spectral index & $\alpha_{\text {inj }}$ & $-0.45 \pm 0.05$ & $-0.52 \pm 0.0$ \\
Source (lobe) age $(\mathrm{Myr})$ & $t_{\mathrm{s.c}}$ & $95 \pm 23$ & $80 \pm 16$ \\
Jet power $\left(\times 10^{45} \mathrm{erg} \mathrm{s}^{-1}\right)$ & $\left\langle Q_{\mathrm{j}}\right\rangle$ & $1.1 \pm 0.1$ & $1.1 \pm 0.1$ \\
Core density $\left(\times 10^{-28} \mathrm{~g} \mathrm{~cm}^{-3}\right)$ & $\left\langle\rho_{0}\right\rangle$ & $4.7 \pm 0.4$ & $4.7 \pm 0.4$ \\
Slope of ambient density distribution & $\beta_{\text {s.c. }}$ & 1.431 & 1.613 \\
External density $\left(\times 10^{-31} \mathrm{~g} \mathrm{~cm}^{-3}\right)$ & $\rho(D)$ & $2.8 \pm 1.1$ & $1.4 \pm 0.7$ \\
Lobe pressure $\left(\times 10^{-14} \mathrm{dyn} \mathrm{cm}^{-2}\right)$ & $p_{\mathrm{lb}}$ & $3.0 \pm 0.1$ & $3.1 \pm 0.1$ \\
Minimum energy magnetic field $(\mu \mathrm{G})$ & $B_{\mathrm{me}}$ & $1.0 \pm 0.2$ & $1.0 \pm 0.2$ \\
Longitudinal expansion speed & $v_{\mathrm{h}} / c$ & $0.05 \pm 0.02$ & $0.06 \pm 0.02$ \\
\hline
\end{tabular}

$\sim 2.2 \mathrm{rad} \mathrm{m}^{-2}$ (on a scale of $\sim 1^{\circ}$ ). As the GRM map has a resolution of $\sim 1^{\circ}$, which is the typical spacing of extragalactic sources in the Taylor et al. (2009) catalogue, it cannot be used to probe RM variations on smaller scales. The true GRM variation on smaller scales at this location is unknown, but RM structure function analyses for GRM variations at high Galactic latitudes have probed scales smaller than $1^{\circ}$ in both observations (e.g. Mao et al. 2010; Stil et al. 2011) and simulations (e.g. Sun \& Reich 2009). In particular, using the results from Stil et al. (2011), we find that GRM variations ranging from approximately $3 \mathrm{rad} \mathrm{m}^{-2}$ to $13 \mathrm{rad} \mathrm{m}^{-2}$ are possible on angular scales of $\sim 11^{\prime}$, depending on the highly uncertain slope of the RM structure function on angular scales less than $1^{\circ}$.

Better estimates of the GRM are required to reliably remove the GRM and its variation across the extent of $\mathrm{J} 1235+5317$.

\subsection{Local environment RM contribution}

The hot gas in rich groups and clusters is known to be magnetised from observations of synchrotron radio halos and relics, as well as Faraday rotation observations of embedded and background radio sources (see Carilli \& Taylor 2002, and references therein). For radio galaxy lobes that have not expanded significantly beyond their host galaxy or cluster/group environment, the Laing-Garrington effect is often present (Laing 1988; Garrington et al. 1988; Garrington \& Conway 1991). This is where the polarised emission from the counter-lobe travels through a greater amount of magnetoionic material and thus incurs a larger amount of Faraday depolarisation. However, as the lobes of $\mathrm{J} 1235+5317$ are expected to be orientated close to the plane of the sky and extend well outside the influence of the group/cluster environment, the Laing-Garrington effect is not expected to be strong (e.g. Laing \& Bridle 2014). Additionally, if the faint collimated emission SE of the host is indeed a jet, then the larger amount of depolarisation towards the SE lobe is opposite to that expected for the Laing-Garrington effect.

Models of the variations in RM across radio galaxies in groups and clusters are typically constructed assuming turbulent magnetic field fluctuations over a range of scales embedded in a spherically-symmetric gas halo whose radial density profile is derived from X-ray observations (e.g. Guidetti et al. 2008). For J1235+5317 we do not have X-ray data to constrain the properties of the hot gas environment, although it is likely that the red-sequence host galaxy is close to the centre of a poor cluster (Hao et al. 2010). Therefore, we attempt to estimate the required density and field strength to selfconsistently explain the mean RM and depolarisation (e.g. 
Murgia et al. 2004), for a single-scale model of a randomly orientated field structure (Felten et al. 1996). In reality, the magnetic field will fluctuate on a range of scales, from an inner scale to an outer scale (Enßlin \& Vogt 2003), a single-scale model can provide a reasonable approximation to the RM variations if the scale length is interpreted as the correlation length of the magnetic field (see Murgia et al. 2004, Sect. 4.4 for details).

An appropriate gas density profile, $n(r)$, for a galaxy group or cluster is a "beta-profile", where $n(r)=n_{0}\left(1+r^{2} / r_{c}^{2}\right)^{-3 \beta / 2}$. We assume that the magnetic field strength scales linearly with the gas density, $B(r)=B_{0} n(r) / n_{0}$, where $B_{0}$ is the central magnetic field strength (e.g. Dolag et al. 2001; Laing et al. 2006; Vacca et al. 2012; Govoni et al. 2017). Values of $n_{0} \sim$ $10^{-3} \mathrm{~cm}^{-3}, r_{c} \sim 100 \mathrm{kpc}$ and $\beta \sim 0.5$ are not unreasonable for a poor cluster (e.g. Laing et al. 2008; Bonafede et al. 2010; Guidetti et al. 2012). The choice of these parameters is arbitrary given our limited information about the environment of the host galaxy (Sect. 1) but we use them simply as a plausible example. Following (Murgia et al. 2004, Eq. (15)), we find a Faraday dispersion of $\sigma_{\mathrm{RM}} \sim 0.1 \mathrm{rad} \mathrm{m}^{-2}$ at $r \sim 1.5 \mathrm{Mpc}$ requires $B_{0} \sim 5 \mu \mathrm{G}$ with a magnetic field correlation length of $\sim 25 \mathrm{kpc}$. This implies an ambient density of $\sim 1.7 \times 10^{-5} \mathrm{~cm}^{-3}$ and field strength $B \sim$ $0.09 \mu \mathrm{G}$ at the location of the hotspots ${ }^{7}$. Using these values and a large outer scale for the magnetic field fluctuations of $500 \mathrm{kpc}$ (Vacca et al. 2010) gives a mean $|\mathrm{RM}|$ of $\sim 0.4 \mathrm{rad} \mathrm{m}^{-2}$. Therefore, while we can reasonably explain $\sigma_{\mathrm{RM}} \sim 0.1 \mathrm{rad} \mathrm{m}^{-2}$ at $r \sim 1.5 \mathrm{Mpc}$, we cannot self-consistently explain the large mean $\mathrm{RM}$ excess of $\sim 2.5 \mathrm{rad} \mathrm{m}^{-2}$, even for a large outer scale of turbulence in the magnetic field power spectrum (Enßlin \& Vogt 2003; Murgia et al. 2004). Note that the outer scale is mainly responsible for the observed mean RM and the inner scale for the value of $\sigma_{\mathrm{RM}}$. We used a large outer scale here to show that this model cannot self-consistently explain both $\sigma_{\mathrm{RM}}$ and the mean RM.

Draping of the ambient field in addition to compression of the ambient magnetoionic gas could enhance the mean RM near the surface of the lobes (Guidetti et al. 2011, 2012), and may also help explain the higher depolarisation of $\sigma_{\mathrm{RM}} \gtrsim$ $0.15 \mathrm{rad} \mathrm{m}^{-2}$ at the location of the SE hotspot. Enhancements in the field strength and gas density by factors of 4 over a path length of $\sim 50 \mathrm{kpc}$ outside the lobes could produce an additional $|\mathrm{RM}|$ of $\sim 0.5 \mathrm{rad} \mathrm{m}^{-2}$. More sensitive observations at high angular resolution are required to determine if such ordered field structures are indeed present.

We note that the external gas density used here is two orders of magnitude higher than estimated from the dynamical modelling. This means that either the observed depolarisation does not occur in the external medium local to the source or that the dynamical modelling is severely underestimating the external density. Such low density gas may be challenging to detect in X-rays, but extrapolation of an $\mathrm{X}$-ray profile from the inner region would be very instructive. In general, comparison with simulations of the propagation of large scale jets within a realistic cosmological environment may provide the best avenue for progress in this area (e.g. Huarte-Espinosa et al. 2011; Hardcastle \& Krause 2014; Turner \& Shabala 2015; English et al. 2016; Vazza et al. 2017).

\footnotetext{
For comparison, using a simple model with a constant electron number density of $n_{\mathrm{e}} \sim 10^{-5} \mathrm{~cm}^{-3}$ and constant magnetic field strength of $B_{\|} \sim 0.1 \mu \mathrm{G}$, with a magnetic field reversal scale of $l \sim 20 \mathrm{kpc}$ over a total path length of $L \sim 1 \mathrm{Mpc}$ gives $\sigma_{\mathrm{RM}} \sim 0.81 n_{\mathrm{e}} B_{\|} \sqrt{l L} \sim$ $0.1 \mathrm{rad} \mathrm{m}^{-2}$.
}

\subsection{Internal Faraday depolarisation}

Our observations are insensitive to polarised emission from RM structures broader than $\sim 1 \mathrm{rad} \mathrm{m}^{-2}$ (Sect. 2.2). Therefore, the large amounts of internal Faraday rotation required to explain the mean RM excess are ruled out. However, it is worth considering if the small amount of Faraday depolarisation $\left(\sigma_{\mathrm{RM}} \sim\right.$ $0.1 \mathrm{rad} \mathrm{m}^{-2}$ ) can be explained by Faraday rotating material mixed with the synchrotron emitting material in the lobes.

One of the most commonly used magnetic field models for the lobes of extragalactic sources is one where the field is highly tangled on small scales, with the observed appreciable degrees of polarisation produced due to stretching and compression (Laing 1980). Given the equipartition magnetic field strength of $\sim 1 \mu \mathrm{G}$ within the lobes (Sect. 3.4), and as an illustrative example, we choose a thermal gas density internal to the lobes of $n_{\mathrm{e}} \sim 10^{-5} \mathrm{~cm}^{-3}$, with 500 field reversals through a lobe depth of $\sim 500 \mathrm{kpc}$, to produce $\sigma_{\mathrm{RM}} \sim 0.1 \mathrm{rad} \mathrm{m}^{-2}$ (using Eq. (1) and assuming $B_{\|}=B / \sqrt{3}$ ). Observations at even lower frequencies would be required to resolve a Faraday depth width of $0.1 \mathrm{rad} \mathrm{m}^{-2}$ in the Faraday spectrum (e.g. using LOFAR observations down to at least $30 \mathrm{MHz}$, in combination with the data in this paper). In addition, broadband polarisation modelling would be needed to distinguish between internal and external Faraday depolarisation scenarios (e.g. Anderson et al. 2018; O'Sullivan et al. 2018). Using the LOFAR international baselines to obtain sub-arcsecond resolution would further enhance the ability to isolate different contributions by resolving the external RM variations across the emission region.

For now, we can assess the likelihood of this scenario in terms of the implied energetics. For expected internal thermal gas temperatures of $\gtrsim 10 \mathrm{keV}$ (Gitti et al. 2007), the lobe thermal gas pressure is $p_{\text {th }} \sim 2 n_{\mathrm{e}} k T \sim 3 \times 10^{-13} \mathrm{dyn} \mathrm{cm}^{-2}$, which is an order of magnitude larger than the pressure from the synchrotron-emitting plasma in the lobes ( $p_{\mathrm{lb}}$ in Table 3). This is inconsistent with expectations from studies of other FRII lobes (Croston et al. 2005; Ineson et al. 2017), and thus unlikely, unless the internal thermal gas is much cooler than assumed here.

\subsection{RM contribution from large-scale structure}

Significant asymmetries in the magnetoionic material in the foreground IGM, far from the local source environment, could also contribute to the observed mean RM difference between the lobes. Such variations could be caused by the magnetised component of the large scale structure (LSS) at low redshift, as Ryu et al. (2008), Cho \& Ryu (2009) and Akahori \& Ryu (2010) predict a root-mean-square RM $\left(\mathrm{RM}_{\mathrm{rms}}\right)$ through LSS filaments of order $1 \mathrm{rad} \mathrm{m}^{-2}$. In our case, the polarised emission of one lobe needs to pass through more foreground filaments than the other to explain the observed RM difference of $2.5 \mathrm{rad} \mathrm{m}^{-2}$. Therefore, information is required on the location of LSS filaments with respect to the lines of sight probed by the polarised emission from the lobes of $\mathrm{J} 1235+5317$.

\subsubsection{Location of large-scale structure filaments}

The catalogue of Chen et al. $(2015,2016)$ provides a cosmic filament reconstruction from the SDSS data for 130 redshift slices in the range $0.05<z<0.7$. In Fig. 6 , we plot the location of the filaments that are in the foreground of $\mathrm{J} 1235+5317$ (i.e. at $z<0.34)$. There are five filaments identified in different foreground redshift slices that pass through the field. We assign a thickness of $1 \mathrm{Mpc}$ to each filament (Vazza et al. 2015b) to 


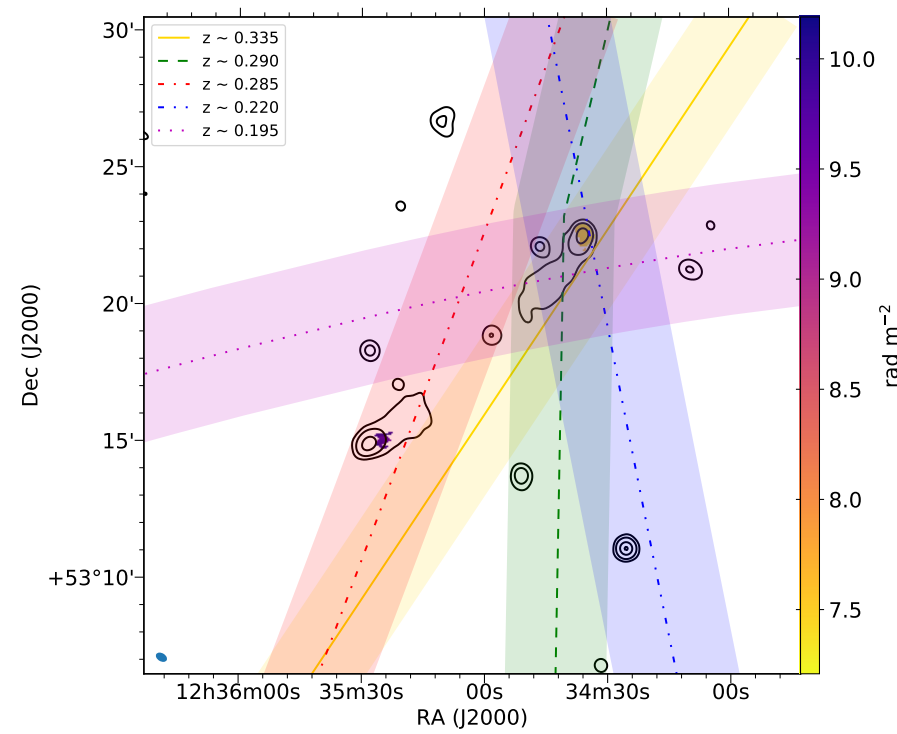

Fig. 6. Location of foreground large-scale-structure filaments (lines) in relation to the background radio galaxy (contours) and its Faraday rotation measure (colour scale), as described in Fig. 3. The width of the lines corresponds to $\sim 1 \mathrm{Mpc}$ at the redshift of the filament.

determine which filaments most likely intersect lines of sight towards the polarised lobes (Fig. 6). For a thickness of $1 \mathrm{Mpc}$, there are four filaments that cover the NW lobe and one filament that covers the SE lobe. Therefore, we estimate that there is an excess of three filaments covering the NW lobe. Considering different filament thicknesses results in different numbers of filaments covering each lobe, with an excess of filaments covering the NW lobe remaining for filaments up to a thickness of $\sim 3.8 \mathrm{Mpc}$ (i.e. the thickness above which the same number of filaments cover both lobes). In light of this result, we consider if the RM difference between the lobes can be explained by magnetised gas in these filaments. We note that there is no evidence of an individual intervening galaxy in the SDSS images that could explain the RM difference.

\subsubsection{Magnetic field stength in filaments}

To explain the RM difference between the lobes, an RM excess of $-2.5 \mathrm{rad} \mathrm{m}^{-2}$ must be provided by the three extra filaments covering the NW lobe. Simulations suggest that the electron number density of LSS filaments can vary from $10^{-6}$ to $10^{-4} \mathrm{~cm}^{-3}$ (Cen \& Ostriker 2006; Ryu et al. 2008; Cho \& Ryu 2009; Akahori \& Ryu 2010; Vazza et al. 2015b), thus we adopt a mean electron density of $10^{-5} \mathrm{~cm}^{-3}$. Akahori \& Ryu (2011) found a peak in the RM power spectrum, due to their simulated IGMF in filaments, on scales corresponding to a proper length of $\sim 3 \mathrm{Mpc}$, which they expect to correspond to the typical line-ofsight path through LSS filaments. Therefore, using a path length $(L)$ of $3 \mathrm{Mpc}$ and a coherence length $(l)$ of $300 \mathrm{kpc}$ (Cho \& Ryu $2009)$ leads to a magnetic field strength in the filaments $\left(B_{\mathrm{LSS}}\right)$ of approximately

$B_{\mathrm{LSS}} \sim 0.3\left(\frac{n_{\mathrm{e}}}{10^{-5} \mathrm{~cm}^{-3}}\right)^{-1}\left(\frac{L}{3(3 \mathrm{Mpc})} \frac{l}{300 \mathrm{kpc}}\right)^{-1 / 2} \mu \mathrm{G}$,

for $B_{\|}=B_{\mathrm{LSS}} / \sqrt{3}$. This estimate of the density-weighted IGMF strength of $\sim 0.3 \mu \mathrm{G}$ has significant uncertainty given our limited knowledge of the particle number density of the gas in these filaments, as well as the observationally unconstrained coherence length of the field and the path length though each filament. Furthermore, this estimate cannot be treated as an upper limit as a large Galactic RM variation across the source (Sect. 4.1) could make the difference in RM between the lobes even larger (since the RM can be positive or negative). Furthermore, much larger RM variations are observed across radio relics which cannot be explained by Galactic RM variations, indicating the presence of large scale ordered fields in the outskirts of galaxy clusters (e.g. Kierdorf et al. 2017; Loi et al. 2017).

Therefore, a better approach may be to compare directly with cosmological simulations of the RM contribution from such LSS filaments. These simulations suggest that the magnetic field strength in filaments could range somewhere from $\sim 1$ to $100 \mathrm{nG}$ (e.g. Vazza et al. 2015b). Early hydrodynamic simulations by Ryu et al. (2008) used a prescription to produce magnetic fields from the kinetic energy of turbulent gas flows (guided by expectations from small-scale magnetic dynamo simulations), which produced average IGMF strengths of $\sim 10 \mathrm{nG}$. Subsequent work by Cho \& Ryu (2009) and Akahori \& Ryu (2010, 2011), using the results of these simulations, provided estimates of the "typical" RM contribution from LSS filaments. The most relevant number for Faraday rotation is the gas density $(\rho)$ weighted average of the strength of the magnetic field through the filaments, i.e. $\left\langle(\rho B)^{2}\right\rangle^{1 / 2} /\left\langle\rho^{2}\right\rangle^{1 / 2}$, which gave a few $\times 0.1 \mu \mathrm{G}$ in the above simulations. From this, it was found that the root-mean-square $\mathrm{RM}\left(\mathrm{RM}_{\mathrm{rms}}\right)$ through the filaments scales with the number of filaments $\left(N_{\mathrm{f}}\right)$ as $\mathrm{RM}_{\mathrm{rms}} \sim 1.5 N_{\mathrm{f}}^{1 / 2} \mathrm{rad} \mathrm{m}^{-2}$, up to a saturation point that corresponds to $\sim 25$ filaments for $z>1$. In the case of three filaments, the predicted $\mathrm{RM}_{\mathrm{rms}} \sim 2.6 \mathrm{rad} \mathrm{m}^{-2}$, which is consistent with our observations (where we have an RM difference of $2.5 \mathrm{rad} \mathrm{m}^{-2}$ between only two lines of sight, in which one passes though three additional filaments). Therefore, it can be argued that our results are consistent with the expected Faraday rotation signature from an average magnetic field strength in LSS filaments of $\sim 10 \mathrm{nG}$.

We further investigated the above findings by direct comparison with recent MHD cosmological simulations, as described in Vazza et al. (2014). In particular, we analysed the RM distribution in the warm-hot gas simulated in a cosmic volume of $50^{3} \mathrm{Mpc}^{3}$, at a spatial resolution of $20 \mathrm{kpc}$ (comoving). To better compare with our observations, we generated a long integration cone for this volume, stacking several randomly oriented, mirrored replicas of the volume, covering the comoving distance out to $z=0.34$. In this way, we could measure the probability of having a contribution as large as $2.5 \mathrm{rad} \mathrm{m}^{-2}$ from LSS filaments for the $\mathrm{J} 1235+5317$ observations at $z=0.34$. We found that this occured in only $5 \%$ of cases, for typical magnetisation values of $\sim 10-50 \mathrm{nG}$, amplified from an initial magnetic field strength of $1 \mathrm{nG}$, which was seeded at an early cosmological epoch and is in line with the upper limits given by the Planck satellite (Planck Collaboration XIX 2016). The probability was negligible for a significantly smaller seed field of $0.1 \mathrm{nG}$.

Lower limits on the primordial field strength of $\sim 10^{-16} \mathrm{G}$ (Neronov \& Vovk 2010) and $\sim 10^{-20} \mathrm{G}$ (Takahashi et al. 2013) imply that the true value may indeed be much lower. However, this is not the only possible scenario, as the LSS can be magnetised by a more "astrophysical" mechanism, such as galaxy feedback (e.g. Vazza et al. 2017, for a recent review), or produced by a more efficient dynamo amplification of primordial fields (Ryu et al. 2008) than is found in current MHD simulations. Therefore, from comparison with the MHD simulations, we consider it unlikely that the true RM contribution from the IGMF is as large as $2.5 \mathrm{rad} \mathrm{m}^{-2}$, and that the observed RM excess 
is possibly dominated by other contributions along the line of sight, such as small scale GRM variations (Sect. 4.1).

\section{Conclusions}

We have presented a linear polarisation and Faraday rotation study of a giant FRII radio galaxy, J1235+5317, using data from the LOFAR Two-Metre Sky Survey (Shimwell et al. 2019). After obtaining the spectroscopic redshift of the host galaxy (SDSS J123501.52+531755.0, $z=0.3448 \pm 0.003$ ), we find that the radio galaxy has a projected linear extent of $3.4 \mathrm{Mpc}$. Both lobes are detected in polarisation with a mean RM difference between the lobes of $2.5 \pm 0.1 \mathrm{rad} \mathrm{m}^{-2}$. Small amounts of Faraday depolarisation $\left(\sim 0.1 \mathrm{rad} \mathrm{m}^{-2}\right)$ are also detected. In the absence of direct tracers of the gas density on large scales, we employ dynamical modelling of the advancing hotspots to infer a particle number density of the ambient gas of $n_{\mathrm{e}} \sim 10^{-7} \mathrm{~cm}^{-3}$. This implies that the radio galaxy is expanding into an underdense region of the Universe. However, explaining the observed Faraday depolarisation (that most likely occurs in the environment local to the source) requires $n_{\mathrm{e}} \sim 10^{-5} \mathrm{~cm}^{-3}$ in combination with a turbulent magnetic field strength of $\sim 0.09 \mu \mathrm{G}$ at a distance of $\sim 1.5 \mathrm{Mpc}$ from the host galaxy. Therefore, either the dynamical modelling is underestimating the density of the external medium or the depolarisation does not occur in the local source environment. Simulations of the propagation of FRII jets to large scales within a realistic cosmological environment may help distinguish between these scenarios. In general, the estimated magnetic field strength is unable to account for the observed mean Faraday rotation difference of $2.5 \mathrm{rad} \mathrm{m}^{-2}$ between the two lobes.

Using a catalogue of large scale structure (LSS) filaments in the local universe derived from optical spectroscopic observations, we find an excess of filaments intersecting lines of sight towards the polarised emission of the NW lobe. Assuming that magnetised gas in these LSS filaments is responsible for the RM difference between the lobes, gives a density-weighted magnetic field strength of $0.3 \mu \mathrm{G}$ (assuming $n_{\mathrm{e}} \sim 10^{-5} \mathrm{~cm}^{-3}$, a line-ofsight path length through each filament of $3 \mathrm{Mpc}$, and a magnetic field coherence length of $300 \mathrm{kpc}$ ). However, we find that predictions from cosmological simulations of the RM contribution from LSS filaments gives a low probability ( $5 \%)$ for an $\mathrm{RM}$ contribution as large as $2.5 \mathrm{rad} \mathrm{m}^{-2}$. This probability applies to the case of magnetic fields strengths in the LSS filaments of $10-50 \mathrm{nG}$, which are amplified from primordial magnetic fields close to current upper limits from the CMB of $\sim 1 \mathrm{nG}$ (the probability decreases to $\sim 0 \%$ for weaker fields). Extrapolation of the observed variations in the Milky Way RM to $11^{\prime}$ scales (i.e. the angular size of $\mathrm{J} 1235+5317$ ) indicates that this likely contributes significantly to the mean RM difference, however, further observations are required to obtain better constraints.

In the near future, large samples of RMs from radio galaxies with known redshifts will allow more advanced statistical analysis techniques to be used, such as RM structure function analyses (e.g. Akahori et al. 2014) and cross-correlation with other tracers of LSS (e.g. Stasyszyn et al. 2010; Vernstrom et al. 2017; Brown et al. 2017). This will enable a better separation of the Faraday rotation due to our Galaxy (e.g. Haverkorn et al. 2004; Sun \& Reich 2009; Mao et al. 2010; Stil et al. 2011) from that due to the cosmic web, and put stronger constraints on the strength and structure of the intergalactic magnetic field.

Acknowledgements. This paper is based (in part) on data obtained with the International LOFAR Telescope (ILT) under project codes LC2_038 and LC3_008. LOFAR (van Haarlem et al. 2013) is the Low Frequency Array designed and constructed by ASTRON. It has observing, data processing, and data storage facilities in several countries, that are owned by various parties (each with their own funding sources), and that are collectively operated by the ILT foundation under a joint scientific policy. The ILT resources have benefitted from the following recent major funding sources: CNRS-INSU, Observatoire de Paris and Université d'Orléans, France; BMBF, MIWF-NRW, MPG, Germany; Science Foundation Ireland (SFI), Department of Business, Enterprise and Innovation (DBEI), Ireland; NWO, The Netherlands; The Science and Technology Facilities Council, UK; Ministry of Science and Higher Education, Poland. SPO and MB acknowledge financial support from the Deutsche Forschungsgemeinschaft (DFG) under grant BR2026/23. Part of this work was carried out on the Dutch national e-infrastructure with the support of the SURF Cooperative through grant e-infra $160022 \& 160152$. The LOFAR software and dedicated reduction packages on https://github.com/apmechev/GRID_LRT were deployed on the e-infrastructure by the LOFAR e-infragroup, consisting of J. B. R. Oonk (ASTRON \& Leiden Observatory), A. P. Mechev (Leiden Observatory) and T. Shimwell (ASTRON) with support from N. Danezi (SURFsara) and C. Schrijvers (SURFsara). This research has made use of data analysed using the University of Hertfordshire high-performance computing facility (http: //uhhpc.herts.ac.uk/) and the LOFAR-UK computing facility located at the University of Hertfordshire and supported by STFC [ST/P000096/1]. This research made use of Astropy, a community-developed core Python package for astronomy (Astropy Collaboration 2013) hosted at http: //wWw . astropy . org/, of Matplotlib (Hunter 2007), of APLpy (Robitaille \& Bressert 2012), an open-source astronomical plotting package for Python hosted at http:// aplpy.github.com/, and of TOPCAT, an interactive graphical viewer and editor for tabular data (Taylor et al. 2005). FV acknowledges financial support from the ERC Starting Grant "MAGCOW", no.714196, and the usage of e usage of computational resources on the Piz-Daint supercluster at CSCS-ETHZ (Lugano, Switzerland) under project s701 and s805. Based on observations made with the Nordic Optical Telescope, operated by the Nordic Optical Telescope Scientific Association at the Observatorio del Roque de los Muchachos, La Palma, Spain, of the Instituto de Astrofisica de Canarias. KEH and JPUF acknowledge support by a Project Grant (162948-051) from The Icelandic Research Fund. The Cosmic Dawn Center is funded by the DNRF. RJvW acknowledges support from the ERC Advanced Investigator programme NewClusters 321271 and the VIDI research programme with project number 639.042 .729 , which is financed by the Netherlands Organisation for Scientific Research (NWO). HA benefited from grant DAIP \#66/2018 of Universidad de Guanajuato. KT is partially supported by JSPS KAKENHI Grant Number 16H05999 and 17H01110, MEXT KAKENHI Grant Number 15H05896, and Bilateral Joint Research Projects of JSPS LKM acknowledges support from Oxford Hintze Centre for Astrophysical Surveys which is funded through generous support from the Hintze Family Charitable Foundation. This publication arises from research partly funded by the John Fell Oxford University Press (OUP) Research Fund. SPO thanks A. G. de Bruyn for stimulating discussions on the topic of this paper, and the referee for their helpful comments.

\section{References}

Akahori, T., \& Ryu, D. 2010, ApJ, 723, 476

Akahori, T., \& Ryu, D. 2011, ApJ, 738, 134

Akahori, T., Gaensler, B. M., \& Ryu, D. 2014, ApJ, 790, 123

Akahori, T., Nakanishi, H., Sofue, Y., et al. 2018, PASJ, 70, R2

Anderson, C. S., Gaensler, B. M., Heald, G. H., et al. 2018, ApJ, 855, 41

Astropy Collaboration (Robitaille, T. P., et al.) 2013, A\&A, 558, A33

Banfield, J. K., Wong, O. I., Willett, K. W., et al. 2015, MNRAS, 453, 2326

Beck, A. M., Hanasz, M., Lesch, H., Remus, R.-S., \& Stasyszyn, F. A. 2013, MNRAS, 429, L60

Beck, R., \& Krause, M. 2005, Astron. Nachr., 326, 414

Beck, R., Dobos, L., Budavári, T., Szalay, A. S., \& Csabai, I. 2016, MNRAS, 460, 1371

Becker, R. H., White, R. L., \& Helfand, D. J. 1995, ApJ, 450, 559

Bernardi, G., Greenhill, L. J., Mitchell, D. A., et al. 2013, ApJ, 771, 105

Bilicki, M., Peacock, J. A., Jarrett, T. H., et al. 2016, ApJS, 225, 5

Black, A. R. S., Baum, S. A., Leahy, J. P., et al. 1992, MNRAS, 256, 186

Bonafede, A., Feretti, L., Murgia, M., et al. 2010, A\&A, 513, A30

Bregman, J. N. 2007, ARA\&A, 45, 221

Brentjens, M. A., \& de Bruyn, A. G. 2005, A\&A, 441, 1217

Brescia, M., Cavuoti, S., Longo, G., \& De Stefano, V. 2014, A\&A, 568, A126

Brown, S., Vernstrom, T., Carretti, E., et al. 2017, MNRAS, 468, 4246

Brüggen, M., Ruszkowski, M., Simionescu, A., Hoeft, M., \& Dalla Vecchia, C. 2005, ApJ, 631, L21

Burn, B. J. 1966, MNRAS, 133, 67

Carilli, C. L., \& Taylor, G. B. 2002, ARA\&A, 40, 319

Cen, R., \& Ostriker, J. P. 2006, ApJ, 650, 560 
Chen, Y.-C., Ho, S., Freeman, P. E., Genovese, C. R., \& Wasserman, L. 2015, MNRAS, 454, 1140

Chen, Y.-C., Ho, S., Brinkmann, J., et al. 2016, MNRAS, 461, 3896

Cho, J., \& Ryu, D. 2009, ApJ, 705, L90

Condon, J. J., Cotton, W. D., Greisen, E. W., et al. 1998, AJ, 115, 1693

Croston, J. H., Hardcastle, M. J., Harris, D. E., et al. 2005, ApJ, 626, 733

Dabhade, P., Gaikwad, M., Bagchi, J., et al. 2017, MNRAS, 469, 2886

Davé, R., Cen, R., Ostriker, J. P., et al. 2001, ApJ, 552, 473

de Gasperin, F., Mevius, M., Rafferty, D. A., Intema, H. T., \& Fallows, R. A 2018, A\&A, 615, A179

Dolag, K., Bartelmann, M., \& Lesch, H. 1999, A\&A, 348, 351

Dolag, K., Schindler, S., Govoni, F., \& Feretti, L. 2001, A\&A, 378, 777

Donnert, J., Dolag, K., Lesch, H., \& Müller, E. 2009, MNRAS, 392, 1008

Duncan, K., Sabater, J., Rottgering, H., et al. 2019, A\&A, 622, A3 (LOFAR SI)

English, W., Hardcastle, M. J., \& Krause, M. G. H. 2016, MNRAS, 461, 2025

Enßlin, T. A., \& Vogt, C. 2003, A\&A, 401, 835

Farnsworth, D., Rudnick, L., \& Brown, S. 2011, AJ, 141, 191

Felten, J. E. 1996, in Clusters, Lensing, and the Future of the Universe, eds. V. Trimble, \& A. Reisenegger, ASP Conf. Ser., 88, 271

Flewelling, H. A., Magnier, E. A., Chambers, K. C., et al. 2016, ArXiv e-prints [arXiv: 1612 . 05243]

Furlanetto, S. R., \& Loeb, A. 2001, ApJ, 556, 619

Garrington, S. T., \& Conway, R. G. 1991, MNRAS, 250, 198

Garrington, S. T., Leahy, J. P., Conway, R. G., \& Laing, R. A. 1988, Nature, 331, 147

George, S. J., Stil, J. M., \& Keller, B. W. 2012, PASA, 29, 214

Gitti, M., McNamara, B. R., Nulsen, P. E. J., \& Wise, M. W. 2007, ApJ, 660, 1118

Govoni, F., Murgia, M., Vacca, V., et al. 2017, A\&A, 603, A122

Gregory, P. C., Scott, W. K., Douglas, K., \& Condon, J. J. 1996, ApJS, 103, 427

Guidetti, D., Murgia, M., Govoni, F., et al. 2008, A\&A, 483, 699

Guidetti, D., Laing, R. A., Bridle, A. H., Parma, P., \& Gregorini, L. 2011 , MNRAS, 413, 2525

Guidetti, D., Laing, R. A., Croston, J. H., Bridle, A. H., \& Parma, P. 2012 MNRAS, 423, 1335

Hales, S. E. G., Masson, C. R., Warner, P. J., \& Baldwin, J. E. 1990, MNRAS, 246,256

Hao, J., McKay, T. A., Koester, B. P., et al. 2010, ApJS, 191, 254

Hardcastle, M. J., \& Krause, M. G. H. 2014, MNRAS, 443, 1482

Hardcastle, M. J., Williams, W. L., Best, P. N., et al. 2019, A\&A, 622, A12 (LOFAR SI)

Haverkorn, M., Gaensler, B. M., McClure-Griffiths, N. M., Dickey, J. M., \& Green, A. J. 2004, ApJ, 609, 776

Heald, G., Braun, R., \& Edmonds, R. 2009, A\&A, 503, 409

Heesen, V., Croston, J. H., Morganti, R., et al. 2018, MNRAS, 474, 5049

Horellou, C., Intema, H. T., Smolčić, V., et al. 2018, A\&A, 620, A19

Huarte-Espinosa, M., Krause, M., \& Alexander, P. 2011, MNRAS, 418, 1621

Hunter, J. D. 2007, Comput. Sci. Eng., 9, 90

Ineson, J., Croston, J. H., Hardcastle, M. J., \& Mingo, B. 2017, MNRAS, 467, 1586

Jelić, V., de Bruyn, A. G., Pandey, V. N., et al. 2015, A\&A, 583, A137

Kierdorf, M., Beck, R., Hoeft, M., et al. 2017, A\&A, 600, A18

Konar, C., Jamrozy, M., Saikia, D. J., \& Machalski, J. 2008, MNRAS, 383, 525

Laing, R. A. 1980, MNRAS, 193, 439

Laing, R. A. 1988, Nature, 331, 149

Laing, R. A., \& Bridle, A. H. 2014, MNRAS, 437, 3405

Laing, R. A., Canvin, J. R., Cotton, W. D., \& Bridle, A. H. 2006, MNRAS, 368, 48

Laing, R. A., Bridle, A. H., Parma, P., \& Murgia, M. 2008, MNRAS, 391, 521

Leahy, J. P., Pooley, G. G., \& Riley, J. M. 1986, MNRAS, 222, 753

Lenc, E., Gaensler, B. M., Sun, X. H., et al. 2016, ApJ, 830, 38

Loi, F., Murgia, M., Govoni, F., et al. 2017, MNRAS, 472, 3605

Longair, M. S., \& Riley, J. M. 1979, MNRAS, 188, 625

Machalski, J., Jamrozy, M., Zola, S., \& Koziel, D. 2006, A\&A, 454, 85

Machalski, J., Chyży, K. T., Stawarz, Ł., \& Kozieł, D. 2007, A\&A, 462, 43

Machalski, J., Kozieł-Wierzbowska, D., Jamrozy, M., \& Saikia, D. J. 2008, ApJ, 679,149

Machalski, J., Jamrozy, M., \& Saikia, D. J. 2009, MNRAS, 395, 812

Machalski, J., Jamrozy, M., Stawarz, Ł., \& Kozieł-Wierzbowska, D. 2011, ApJ, 740,58

Machalski, J., Jamrozy, M., Stawarz, Ł., \& Weżgowiec, M. 2016, A\&A, 595, A46

Mack, K.-H., Klein, U., O’Dea, C. P., Willis, A. G., \& Saripalli, L. 1998, A\&A, 329,431

Malarecki, J. M., Jones, D. H., Saripalli, L., Staveley-Smith, L., \& Subrahmanyan, R. 2015, MNRAS, 449, 955

Mao, S. A., Gaensler, B. M., Haverkorn, M., et al. 2010, ApJ, 714, 1170

Mingo, B., Hardcastle, M. J., Ineson, J., et al. 2017, MNRAS, 470, 2762

Mulcahy, D. D., Horneffer, A., Beck, R., et al. 2014, A\&A, 568, A74
Murgia, M., Govoni, F., Feretti, L., et al. 2004, A\&A, 424, 429

Neld, A., Horellou, C., Mulcahy, D. D., et al. 2018, A\&A, 617, A136

Neronov, A., \& Vovk, I. 2010, Science, 328, 73

Nicastro, F., Kaastra, J., Krongold, Y., et al. 2018, Nature, 558, 406

Offringa, A. R., McKinley, B., Hurley-Walker, et al. 2014, MNRAS, 444, 606

Oppermann, N., Junklewitz, H., Robbers, G., et al. 2012, A\&A, 542, A93

Oppermann, N., Junklewitz, H., Greiner, M., et al. 2015, A\&A, 575, A118

Orrù, E., van Velzen, S., Pizzo, R. F., et al. 2015, A\&A, 584, A112

O'Sullivan, S. P., Lenc, E., Anderson, C. S., Gaensler, B. M., \& Murphy, T. 2018, MNRAS, 475, 4263

Pedani, M., \& Grueff, G. 1999, A\&A, 350, 368

Pirya, A., Saikia, D. J., Singh, M., \& Chandola, H. C. 2012, MNRAS, 426, 758

Planck Collaboration XIII. 2016, A\&A, 594, A13

Planck Collaboration XIX. 2016, A\&A, 594, A19

Planck Collaboration XXII. 2016, A\&A, 594, A22

Rengelink, R. B., Tang, Y., de Bruyn, A. G., et al. 1997, A\&AS, 124, 259

Riley, J. M. W., Waldram, E. M., \& Riley, J. M. 1999, MNRAS, 306, 31

Riseley, C. J., Lenc, E., Van Eck, C. L., et al. 2018, PASA, accepted [arXiv:1809. 09327]

Robitaille, T., \& Bressert, E. 2012, Astrophysics Source Code Library [record ascl:1208.017]

Ryu, D., Kang, H., Cho, J., \& Das, S. 2008, Science, 320, 909

Safouris, V., Subrahmanyan, R., Bicknell, G. V., \& Saripalli, L. 2009, MNRAS, 393, 2

Schoenmakers, A. P., de Bruyn, A. G., Röttgering, H. J. A., \& van der Laan, H. 2001, A\&A, 374, 861

Shimwell, T. W., Röttgering, H. J. A., Best, P. N., et al. 2017, A\&A, 598, A104

Shimwell, T. W., Tasse, C., Hardcastle, M. J., et al. 2019, A\&A, 622, A1 (LOFAR SI)

Sotomayor-Beltran, C., Sobey, C., Hessels, J. W. T., et al. 2013, A\&A, 552, A58

Stasyszyn, F., Nuza, S. E., Dolag, K., Beck, R., \& Donnert, J. 2010, MNRAS, 408, 684

Stil, J. M., Taylor, A. R., \& Sunstrum, C. 2011, ApJ, 726, 4

Subrahmanyan, R., Saripalli, L., Safouris, V., \& Hunstead, R. W. 2008, ApJ, 677, 63

Sun, X. H., \& Reich, W. 2009, A\&A, 507, 1087

Takahashi, K., Mori, M., Ichiki, K., Inoue, S., \& Takami, H. 2013, ApJ, 771, L42

Taylor, M. B. 2005, in Astronomical Data Analysis Software and Systems XIV, eds. P. Shopbell, M. Britton, \& R. Ebert, ASP Conf. Ser., 347, 29

Taylor, A. R., Stil, J. M., \& Sunstrum, C. 2009, ApJ, 702, 1230

Turner, R. J., \& Shabala, S. S. 2015, ApJ, 806, 59

Vacca, V., Murgia, M., Govoni, F., et al. 2010, A\&A, 514, A71

Vacca, V., Murgia, M., Govoni, F., et al. 2012, A\&A, 540, A38

Vacca, V., Oppermann, N., Enßlin, T., et al. 2016, A\&A, 591, A13

Vacca, V., Murgia, M., Govoni, F., et al. 2018, MNRAS, 479, 776

van Diepen, G. e. r., \& Dijkema, T. J. 2011, Astrophysics Source Code Library [record ascl:1804.003]

Van Eck, C. L., Haverkorn, M., Alves, M. I. R., et al. 2018, A\&A, 613, A58 van Haarlem, M. P., Wise, M. W., Gunst, A. W., et al. 2013, A\&A, 556, A2 Vazza, F., Brüggen, M., Gheller, C., \& Wang, P. 2014, MNRAS, 445, 3706

Vazza, F., Ferrari, C., \& Bonafede, A. 2015a, Advancing Astrophysics with the Square Kilometre Array (AASKA14), 97

Vazza, F., Ferrari, C., Brüggen, M., et al. 2015b, A\&A, 580, A119

Vazza, F., Brüggen, M., \& Gheller, C. 2017, CQG, 34, 234001

Vernstrom, T., Gaensler, B. M., Brown, S., Lenc, E., \& Norris, R. P. 2017, MNRAS, 467, 4914

Waldram, E. M., Pooley, G. G., Davies, M. L., Grainge, K. J. B., \& Scott, P. F. 2010, MNRAS, 404, 1005

Williams, W. L., Hardcastle, M. J., Best, P. N., et al. 2019, A\&A, 622, A2 (LOFAR SI)

Worrall, D. M., \& Birkinshaw, M. 2006, in Lect. Notes Phys., ed. D. Alloin (Berlin: Springer Verlag), Physics of Active Galactic Nuclei at all Scales, 693, 39

Xu, Y., Kronberg, P. P., Habib, S., \& Dufton, Q. W. 2006, ApJ, 637, 19

Zweibel, E. G. 2006, Astron. Nachr., 327, 505

1 Hamburger Sternwarte, Universität Hamburg, Gojenbergsweg 112, 21029 Hamburg, Germany

e-mail: shane@hs . uni-hamburg.de

2 Astronomical Observatory, Jagiellonian University, ul. Orla 171, Kraków 30-244, Poland

3 Department of Physics and Astronomy, University of Calgary, Calgary, Alberta T2N 1N4, Canada

4 CSIRO Astronomy and Space Science, PO Box 1130, Bentley, WA 6102, Australia 
5 The Cosmic Dawn Center, Niels Bohr Institute, University of Copenhagen, Juliane Maries Vej 30, 2100 Copenhagen Ø, Denmark

${ }^{6}$ Centre for Astrophysics and Cosmology, Science Institute, University of Iceland, Dunhagi 5, 107 Reykjavík, Iceland

7 Dark Cosmology Centre, Niels Bohr Institute, University of Copenhagen, Juliane Maries Vej 30, 2100 Copenhagen Ø, Denmark

8 INAF - Osservatorio Astronomico di Cagliari, Via della Scienza 5, 09047 Selargius (CA), Italy

9 Centre for Astrophysics Research, School of Physics, Astronomy and Mathematics, University of Hertfordshire, College Lane, Hatfield AL10 9AB, UK

10 ASTRON, The Netherlands Institute for Radio Astronomy, Postbus 2, 7990 AA Dwingeloo, The Netherlands

11 Leiden Observatory, Leiden University, PO Box 9513, 2300 RA Leiden, The Netherlands

12 GEPI \& USN, Observatoire de Paris, Université PSL, CNRS, 5 Place Jules Janssen, 92190 Meudon, France

13 Department of Physics \& Electronics, Rhodes University, PO Box 94, Grahamstown 6140, South Africa

14 Dipartimento di Fisica e Astronomia, Universitá di Bologna, Via Gobetti 93/2, 40121 Bologna, Italy
15 Departamento de Astronomía, DCNE, Universidad de Guanajuato, Guanajuato, Mexico

${ }^{16}$ HH Wills Physics Laboratory, University of Bristol, Tyndall Avenue Bristol BS8 1TL, UK

17 Department of Astrophysics/IMAPP, Radboud University Nijmegen, PO Box 9010, 6500 GL Nijmegen, the Netherlands

18 Dept. of Space, Earth and Environment, Chalmers University of Technology, Onsala Space Observatory, 43992 Onsala, Sweden

19 INAF - Istituto di Radioastronomia, via P. Gobetti 101, 40129 Bologna, Italy

20 GFZ German Research Centre for Geosciences, Telegrafenberg, 14473 Potsdam, Germany

21 Max-Planck-Institut für Radioastronomie, Auf dem Hügel 69, 53121, Bonn, Germany

22 Department of Physics, Kumamoto University, Kumamoto 8608555, Japan

23 Dunlap Institute for Astronomy and Astrophysics University of Toronto, Toronto, ON M5S 3H4, Canada

24 Astrophysics, University of Oxford, Denys Wilkinson Building, Keble Road, Oxford OX1 3RH, UK 